
\title{
$\begin{array}{ll}\text { Research Square } & \text { They should not be considered conclusive, used to inform clinical practice, } \\ \text { or referenced by the media as validated information. }\end{array}$
}

\section{Quantitative Assessment of Organic And Inorganic Contaminants In Charcoal Applied for Food Processing}

Zbigniew Jelonek ( $\square$ zjelonek@us.edu.pl )

University of Silesia in Katowice

Monika Fabiańska

University of Silesia in Katowice

Iwona Jelonek

University of Silesia in Katowice

\section{Research Article}

Keywords: gas chromatography - mass spectrometry, atomic absorption spectroscopy

Posted Date: April 1st, 2021

DOI: https://doi.org/10.21203/rs.3.rs-369239/v1

License: () (i) This work is licensed under a Creative Commons Attribution 4.0 International License. Read Full License 


\section{Abstract}

Thirty-one batches of commercial charcoal from various regions of Poland and Germany were tested for the presence of twenty toxic elements and polycyclic aromatic hydrocarbons (PAHs) using gas chromatography - mass spectrometry (GC-MS). Elements that are toxic to living organisms were determined using atomic absorption spectroscopy (AAS). They were classified as elements representing a very high degree of hazard (As, $\mathrm{Cd}, \mathrm{Cu}, \mathrm{Hg}$, and $\mathrm{Pb})$, high degree of hazard ( $\mathrm{Zn}, \mathrm{Ba}, \mathrm{Cr}, \mathrm{Mn}$, and $\mathrm{Mo}$ ), moderate degree of hazard (Co, Ni, Sn, and Te), and a low degree of hazard for living organisms and the environment (Ag, Bi, $\mathrm{Ce}, \mathrm{Se}, \mathrm{Sr}$, and $\mathrm{Zr}$ ). When it comes to the most toxic elements, the highest concentration in the whole tested material was recorded for Cu. In addition, considerable amounts of $\mathrm{Ba}, \mathrm{Mn}$, and $\mathrm{Sr}$, i.e., elements representing high or moderate degree of hazard, were found in the tested charcoals. Moreover, all charcoals contained a wide range of PAHs, from naphthalene to benzo(ghi)perylene, with concentrations in the range between 12.55 and $3554.11 \mathrm{ng} / \mathrm{g}$ of charcoal. In total, 25 unsubstituted PAHs were identified in the charcoal extracts. PAHs distributions were dominated by 5-ring PAHs. The results indicate the high carcinogenicity with $\sum$ PAHcarc/ $\sum$ PAHtot close to 1, as well as high TEQ and MEQ values. Thus, prolonged exposure to charcoal and charcoal dust might cause serious health problems. This applies to employees actively involved in the production and transport of charcoal, and, to a lesser extent, also to users of this fuel.

\section{Introduction}

Charcoal has been used at least since 50,000 years B.C., which is confirmed by the study of wood tar used to attach stone points to spears. It indicates the first conscious attempts to produce charcoal using the backfill method, i.e., through a partial dry wood distillation process under the earth cover limiting oxygen access. In this way, small amounts of tar, an excellent adhesive and preservative product, were obtained as a by-product ${ }^{1}$. The use of high temperature for meat processing resulted in a more digestible diet, more extended storage of processed food, and allowed us to obtain tar ${ }^{2}$. The demand for charcoal production increased in the Bronze and Iron Age when it facilitated everyday metal objects and weapons production. The addition of carbon to iron during the smelting of ore in charcoal-fired furnaces became the basis of modern civilization ${ }^{3}$. At the end of the 19th century, the industry gradually moved away from charcoal towards fossil fuels and petroleum components. The renaissance of charcoal as fuel in its original form occurred in the 1940 s. Grill grates for meat hung over a fire on a semicircular bowl filled with fuel (charcoal) were quickly adopted worldwide 4 . Grilling has become a favorite form of food preparation during family gatherings and outdoor activities.

In the 1980s of the twentieth century, researchers focused on the quality and possible harmfulness of grilled food, e.g., carcinogenic substances in grilled meat $^{5-6-7}$. It was found that polycyclic aromatic hydrocarbons (PAHs) in it primarily come from thermal treatment of meat (fat and meat grilled directly over a heated surface) and gaseous emissions from charcoal combustion. Medical observations confirmed that PAHs contribute to many civilization and occupational diseases in modern society ${ }^{8}$.

The exposure to fuels used for heat food treatment such as charcoal can pose many risks, not only limited to charcoal combustion as a barbecue fuel. Risks may arise when pouring, packing, transporting, and storing barbecue fuels characterized by low density, which promotes their easy crushing and dusting. Employees in production plants and broadly defined consumers, both adults and children, are exposed to fine charcoal dust emission. The knowledge of carcinogenic compounds and selected harmful chemical elements contents in grill fuels is as important as their combustion on people, food products, and the environment.

The research presented here aims to assess PAHs' and carcinogenic elements concentration levels in charcoals intended for grill devices. Up to our knowledge, charcoal used for cooking has not been examined on a large scale for these components' presence. Researchers mainly focused on analyzing thermally processed food products or evaluating the gases emitted during charcoal combustion. This study of grill fuels shows that harmful compounds are already present in the fuel itself. However, their contents vary from one fuel to another. Thus, it possible to limit the charcoal users' exposure to these compounds by eliminating the most contaminated charcoals if their composition is known.

\section{Methods}

The individual charcoals were quartered, then each fourth part of each sample was sent to the accredited laboratory, Bureau Veritas Commodities. Canada Ltd." The analysis was performed in accordance with the applied methodology specified under the analytical code MA250 and AQ200. The concentration of the following elements was determined: $\mathrm{Mo}, \mathrm{Cu}, \mathrm{Pb}, \mathrm{Zn}, \mathrm{Ni}, \mathrm{Mn}, \mathrm{Sr}, \mathrm{Cd}, \mathrm{Ba}, \mathrm{Ce}, \mathrm{Hg}, \mathrm{Co}, \mathrm{Te}, \mathrm{Zr}, \mathrm{Se}, \mathrm{Cr}, \mathrm{Ag}, \mathrm{As}$, Sn, and Bi. The listed elements were selected on the basis of their toxicity very high, high, medium, low9, and their limit values determined on the basis of PN-EN ISO 17225-1:2014 - 0710, PN-EN ISO 16967:2015 - 0611 and "Hazardous substance fact sheet"12 and presented in Table 1.

Table 1

Limit values of toxic elements in solid fuels (based on Z. Jelonek, 2021).

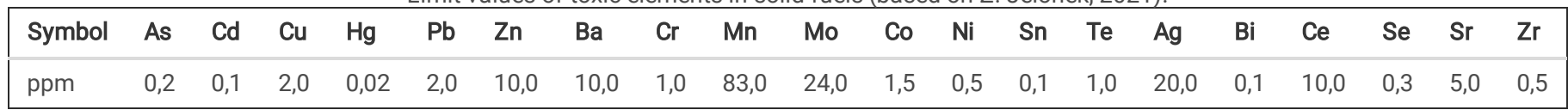

Charcoal was purchased in original commercial packaging with a net unit weight of $2500 \mathrm{~g}$, partially directly from grill fuel producers as a part of cooperation, partially bought in supermarkets. Altogether 31 different charcoals were obtained for the research aims. Charcoal samples were classified according to the method of obtaining material for research and production method, and the location of production. The type of wood used for the production of charcoal was also declared by the grill fuel producers (Table 2). 
Table 2

Production method, production location and type of wood used to produce the charcoal.

\begin{tabular}{|c|c|c|c|c|c|}
\hline \multirow[t]{2}{*}{ Sample } & \multicolumn{3}{|c|}{ Production method* } & \multirow{2}{*}{$\begin{array}{l}\text { Location } \\
\text { Country/voivodeship or region }\end{array}$} & \multirow[t]{2}{*}{ Type of wood used for the production } \\
\hline & Generator & Annealed & Earth pits & & \\
\hline 1 & 2 & 3 & 4 & 5 & 6 \\
\hline W1 & $x$ & & & Poland /West Pomeranian Voivodeship & hornbeam, beech, ash \\
\hline W2 & & $\mathrm{x}$ & & Poland/West Pomeranian Voivodeship & beech, birch \\
\hline W3 & & $\mathrm{x}$ & & Poland/Lublin Voivodeship & hornbeam, beech, ash, oak \\
\hline W4 & no data & & & & \\
\hline W5 & & $x$ & & Poland/Podlaskie Voivodeship & beech \\
\hline W6 & no data & & & & \\
\hline W7 & $\mathrm{X}$ & & & Poland/Podlaskie Voivodeship & hornbeam, beech, ash, oak \\
\hline W8 & & $\mathrm{x}$ & & Poland/Mazovian Voivodeship & hornbeam, beech, ash, oak \\
\hline W9 & & & $\underline{x}$ & Poland/Podlaskie Voivodeship & beech, oak \\
\hline W10 & & $\underline{x}$ & & Poland/Podlaskie Voivodeship & beech \\
\hline W11 & & $\underline{x}$ & & Poland/Greater Poland Voivodeship & beech, oak, ash, hornbeam, \\
\hline W12 & & $\underline{x}$ & & Poland/Lesser Poland & beech, hornbeam, oak, birch \\
\hline W13 & no data & & & & \\
\hline W14 & & & $\underline{x}$ & Poland/Podkarpackie Voivodeship & beech, hornbeam \\
\hline W15 & $\underline{x}$ & & & Poland/Greater Poland Voivodeship & birch, hornbeam, oak, beech \\
\hline W16 & $\underline{x}$ & & & Poland/West Pomeranian Voivodeship & hornbeam, beech, ash, oak \\
\hline W17 & no data & & & & \\
\hline W18 & & $\underline{x}$ & & Poland/West Pomeranian Voivodeship & $\underline{\text { beech, ash }}$ \\
\hline W19 & $\underline{x}$ & & & Poland/Greater Poland Voivodeship & beech, hornbeam, oak, birch \\
\hline W20 & & $\underline{x}$ & & Poland/Mazovian Voivodeship & no data \\
\hline W21 & & $x$ & & Poland/Lesser Poland & beech, hornbeam, oak, birch \\
\hline W22 & & $\underline{x}$ & & Poland/Greater Poland Voivodeship & beech, hornbeam, oak, birch \\
\hline W23 & & $\underline{x}$ & & Poland/Mazovian Voivodeship & mixed deciduous wood \\
\hline W24 & $\underline{x}$ & & & Poland/Kuyavian-Pomeranian Voivodeship & $\underline{\text { mixed deciduous wood }}$ \\
\hline W25 & & $\underline{x}$ & & Poland/Silesian Voivodeship & beech, hornbeam, birch \\
\hline W26 & & $\underline{x}$ & & Poland/Mazovian Voivodeship & beech, hornbeam, oak, birch \\
\hline W27 & & $\underline{x}$ & & Poland/Kuyavian-Pomeranian Voivodeship & no data \\
\hline W28 & & $\underline{x}$ & & Poland/Pomeranian Voivodeship & beech, hornbeam, oak, birch \\
\hline W29 & & $\underline{x}$ & & Germany/Mannheim & beech, hornbeam, oak, birch \\
\hline W30 & & $\underline{x}$ & & Poland/Lesser Poland & hornbeam, birch \\
\hline W31 & & $\mathrm{x}$ & & Poland/Lower Silesian Voivodeship & beech, hornbeam, oak, birch \\
\hline
\end{tabular}

* Charcoal production method

Column 2. Generator (wood is burnt with low airflow in retorts)

Column 3. Annealed (wood is annealed under controlled conditions, usually using modern automated production lines)

Column 4. Earth pits (wood is burnt in an earth pit by arranging wooden logs on burning branches and covering the pit with excavated earth to cut off the air supply) 13

The charcoal was ground (from each package separately) to a fraction of 1-0.5 mm. After averaging, from the material obtained in this way, samples weighing $4 \mathrm{~g}-7 \mathrm{~g}$ were taken. Each of the samples was subjected to the extraction in the Dionex ASE 350 extractor dedicated for rapid extraction under pressure. The extracts were evaporated at room temperature and weighted. Extraction yields are shown in Table 3 . The obtained extracts were derivatized to obtain derivatives with properties enabling their determination using the applied analytical method. To each extract obtained from the samples, $100 \mu \mathrm{l}$ of $\mathrm{N}, \mathrm{O}-$ 
bis (trimethylsilyl) trifluoroacetamide (BSTFA) and $10 \mu \mathrm{l}$ of pyridine were added. Then, the solution was reconstituted up to $1 \mathrm{ml}$ with hexane. The prepared solutions were placed in a laboratory furnace for 3 hours at a temperature of $700 \mathrm{C}$. After removing the extracts from the oven, any fluid losses from individual vials were reconstituted to $1 \mathrm{ml}$ with a solvent (hexane).

The derivatized charcoal extracts were analyzed with an Agilent 7890A gas chromatograph equipped with an HP-35 column coated with a $0.25-\mu m$ stationary phase film, coupled with an Agilent Technology 5975C XL MDS mass spectrometer. The experimental conditions were as follows: carrier gas - He; temperature $-50^{\circ} \mathrm{C}$ (isothermal for $2 \mathrm{~min}$ ); heating rate - up to $175^{\circ} \mathrm{C}$ at $10^{\circ} \mathrm{C} / \mathrm{min}$, to $225^{\circ} \mathrm{C}$ at $6^{\circ} \mathrm{C} / \mathrm{min}$ and, finally, to $300^{\circ} \mathrm{C}$ at $4^{\circ} \mathrm{C} / \mathrm{min}$. The final temperature $\left(300^{\circ} \mathrm{C}\right.$ ) was held for $20 \mathrm{~min}$. The mass spectrometer was operated in the ionization mode (70 eV, full scan) and scanned from 50-650 Da. The compounds were identified by their mass spectra, comparison of peak retention times with those of standard compounds. The data was processed using Hewlett-Packard Chemstation software. Peaks were integrated manually. The quantitative analysis was done based on the 5-point calibration curves for the analytical standards. All standards purity was checked prior to calibration curves preparation in the same analytical conditions as used for the extract analyses. The derivatizing reagents, stock solutions and calibration solutions containing the analytes were stored in the absence of light and under refrigeration at $5^{\circ} \mathrm{C}$.

The linear correlation between the peak areas and PAHs concentrations were checked within the range $0.10-10 \mu \mathrm{g} / \mathrm{mL}$, with correlation coefficients values within the range of $0.997-0.0998$. For quality assurance and quality control (QA/QC) the analysis of each sample-series was accompanied by the analysis of a blank sample in order to assess possible contamination during the analytical procedure. The limits of detection (LODs) were calculated as three times the standard deviation of background peaks in the procedural blanks. LODs values were $2.0 \pm 0.05 \mathrm{ng} / \mathrm{mL}$. Concentrations below LOD were considered as zero for all calculations.

\section{Results}

A quantitative analysis of charcoal samples for the content of elements harmful to humans is shown in Table 3. 
Table 3

Summary of the elemental content of the tested charcoals.

\begin{tabular}{|c|c|c|c|c|c|c|c|c|c|c|c|c|c|c|c|c|c|c|}
\hline Analyte & Wgt & As & $\mathrm{Cd}$ & $\mathrm{Cu}$ & $\mathrm{Hg}$ & $\mathrm{Pb}$ & $\mathrm{Zn}$ & $\mathrm{Ba}$ & $\mathrm{Cr}$ & Mn & Mo & Co & $\mathrm{Ni}$ & Sn & $\mathrm{Te}$ & $\mathrm{Ag}$ & $\mathrm{Bi}$ & $\mathrm{Ce}$ \\
\hline Unit & kg & ppm & ppm & ppm & ppm & ppm & ppm & ppm & ppm & ppm & ppm & ppm & ppm & ppm & ppm & ppm & ppm & ppm \\
\hline MDL & 0.01 & 0.2 & 0.02 & 0.1 & 0.01 & 0.02 & 0.2 & 1 & 1 & 1 & 0.05 & 0.2 & 0.1 & 0.1 & 0.05 & 0.02 & 0.04 & 0.02 \\
\hline W1 & 0.04 & $\begin{array}{l}< \\
0.2\end{array}$ & 0.02 & 4.5 & $\begin{array}{l}<.01 \\
0.01\end{array}$ & 1.58 & 7.6 & 98 & 1 & 32.0 & 1.24 & 0.3 & 0.9 & $\begin{array}{l}< \\
0.1\end{array}$ & 0.88 & $\begin{array}{l}< \\
0.02\end{array}$ & $\hat{0}_{0.04}$ & 1.73 \\
\hline W2 & 0.06 & $\begin{array}{l}<.2 \\
0.2\end{array}$ & 0.02 & 3.8 & $\begin{array}{l}< \\
0.01\end{array}$ & 0.36 & 7.7 & 96 & $<1$ & 124.0 & 0.24 & 0.2 & 5.0 & $\dot{0}_{0.1}$ & 0.20 & $\begin{array}{l}< \\
0.02\end{array}$ & $\hat{0}_{0.04}$ & 0.29 \\
\hline W3 & 0.04 & $\begin{array}{l}< \\
0.2\end{array}$ & 0.02 & 4.0 & $\begin{array}{l}< \\
0.01\end{array}$ & 0.26 & 6.0 & 157 & $<1$ & 240.0 & 0.10 & 0.2 & 2.0 & $\begin{array}{l}< \\
0.1\end{array}$ & 0.16 & $\begin{array}{l}< \\
0.02\end{array}$ & $\begin{array}{l}< \\
0.04\end{array}$ & 0.10 \\
\hline W4 & 0.06 & 0.2 & 0.09 & 4.1 & 0.02 & 0.92 & 31.8 & 125 & 1 & 218.0 & 0.08 & 0.2 & 4.8 & $\begin{array}{l}< \\
0.1\end{array}$ & 0.30 & 0.02 & $\hat{0}_{0.04}$ & 0.06 \\
\hline W5 & 0.06 & $\begin{array}{l}<.2 \\
0.2\end{array}$ & 0.03 & 5.8 & $\begin{array}{l}<.01 \\
0.01\end{array}$ & 0.27 & 6.6 & 57 & $<1$ & 717.0 & 0.09 & 0.2 & 3.1 & $\dot{0}_{0.1}$ & 0.09 & 0.24 & $\hat{0}_{0.04}$ & 0.13 \\
\hline W6 & 0.05 & $\begin{array}{l}< \\
0.2\end{array}$ & 0.07 & 4.2 & $\begin{array}{l}<.01 \\
0.01\end{array}$ & 0.81 & 26.0 & 109 & 1 & 231.0 & 0.08 & 0.2 & 4.6 & $\begin{array}{l}< \\
0.1\end{array}$ & 0.18 & $\begin{array}{l}< \\
0.02\end{array}$ & $\hat{0}_{0.04}$ & 0.09 \\
\hline W7 & 0.05 & 0.3 & 0.05 & 3.7 & $\begin{array}{l}<.01 \\
0.01\end{array}$ & 0.38 & 8.4 & 125 & $<1$ & 273.0 & 0.06 & 0.2 & 1.8 & $\begin{array}{l}< \\
0.1\end{array}$ & 0.13 & 0.33 & $\hat{0}_{0.04}$ & 0.08 \\
\hline W8 & 0.05 & $\begin{array}{l}< \\
0.2\end{array}$ & 0.20 & 7.0 & $\begin{array}{l}< \\
0.01\end{array}$ & 1.50 & 47.3 & 44 & $<1$ & 127.0 & 0.07 & $\begin{array}{l}< \\
0.2\end{array}$ & 0.6 & $\dot{0}_{0.1}$ & 0.05 & 0.82 & $\hat{0}_{0.04}$ & 0.06 \\
\hline W9 & 0.05 & 0.3 & 0.09 & 4.0 & $\begin{array}{l}<.01 \\
0.01\end{array}$ & 0.69 & 8.0 & 129 & $<1$ & 220.0 & 0.81 & $\begin{array}{l}<.2 \\
0.2\end{array}$ & 1.0 & $\begin{array}{l}< \\
0.1\end{array}$ & 0.58 & $\begin{array}{l}<.02 \\
0.02\end{array}$ & $\hat{0}_{0.04}$ & 0.59 \\
\hline W10 & 0.05 & 0.3 & 0.23 & 3.4 & $\dot{0}_{0.01}$ & 1.98 & 41.9 & 97 & $<1$ & 1817.0 & 0.05 & 0.5 & 1.0 & $\grave{0}_{0.1}$ & 0.11 & $\begin{array}{l}<.02 \\
0.02\end{array}$ & $\dot{0}_{0.04}$ & 0.08 \\
\hline W11 & 0.04 & $\begin{array}{l}<.2 \\
0.2\end{array}$ & 0.03 & 4.0 & $\begin{array}{l}< \\
0.01\end{array}$ & 0.36 & 4.5 & 44 & $<1$ & 127.0 & 0.05 & $\begin{array}{l}<.2 \\
0.2\end{array}$ & 0.5 & $\dot{ }_{0.1}$ & 0.12 & $\begin{array}{l}<.02 \\
0.02\end{array}$ & $\hat{0}_{0.04}$ & 0.03 \\
\hline W12 & 0.04 & 0.2 & 0.09 & 3.5 & $\begin{array}{l}<.01 \\
0.01\end{array}$ & 1.65 & 23.1 & 47 & $<1$ & 366.0 & 0.09 & $\hat{0}_{0.2}$ & 1.0 & $\begin{array}{l}<.1 \\
0.1\end{array}$ & 0.18 & 0.34 & $\begin{array}{l}<.04 \\
0.04\end{array}$ & 0.18 \\
\hline W13 & 0.04 & 0.2 & 0.09 & 2.4 & $\hat{0}_{0.01}$ & 2.0 & 11.3 & 63 & $<1$ & 183.0 & 0.09 & 0.2 & 0.9 & $\begin{array}{l}< \\
0.1\end{array}$ & 0.29 & $\begin{array}{l}<.02 \\
0.02\end{array}$ & $\hat{0}_{0.04}$ & 0.09 \\
\hline W14 & 0.04 & $\begin{array}{l}<.2 \\
0.2\end{array}$ & 0.04 & 5.1 & $\dot{ }_{0.01}$ & 0.23 & 3.5 & 37 & $<1$ & 76.0 & 0.05 & $\begin{array}{l}<.2 \\
0.2\end{array}$ & 1.5 & $\dot{ }_{0.1}$ & 0.20 & $\begin{array}{l}<.02 \\
0.02\end{array}$ & $\hat{0}_{0.04}$ & 0.09 \\
\hline W15 & 0.04 & $\begin{array}{l}< \\
0.2\end{array}$ & 0.04 & 3.7 & $\begin{array}{l}<.01 \\
0.01\end{array}$ & 0.36 & 9.0 & 46 & $<1$ & 272.0 & 0.07 & 0.4 & 2.4 & $\begin{array}{l}< \\
0.1\end{array}$ & 0.14 & 0.21 & $\begin{array}{l}<.04 \\
0.04\end{array}$ & 0.17 \\
\hline W16 & 0.05 & 0.5 & 0.02 & 4.5 & $\begin{array}{l}< \\
0.01\end{array}$ & 0.65 & 60.2 & 79 & $<1$ & 293.0 & 0.05 & 0.2 & 1.5 & $\dot{0}_{0.1}$ & 0.12 & $\begin{array}{l}<.02 \\
0.02\end{array}$ & $\hat{0}_{0.04}$ & 0.11 \\
\hline W17 & 0.04 & $\begin{array}{l}<.2 \\
0.2\end{array}$ & 0.15 & 2.9 & $\begin{array}{l}<.01 \\
0.01\end{array}$ & 0.97 & 21.6 & 71 & $<1$ & 169.0 & 0.09 & $\begin{array}{l}<.2 \\
0.2\end{array}$ & 1.5 & $\dot{ }_{0.1}$ & 0.16 & $\begin{array}{l}< \\
0.02\end{array}$ & $\dot{0}_{0.04}$ & 0.93 \\
\hline W18 & 0.04 & $\hat{0}_{0.2}$ & 0.02 & 2.7 & $\begin{array}{l}<.01 \\
0.01\end{array}$ & 0.19 & 9.6 & 4 & $<1$ & 8.0 & 0.05 & $\begin{array}{l}<.2 \\
0.2\end{array}$ & 0.3 & $\begin{array}{l}< \\
0.1\end{array}$ & 0.10 & $\begin{array}{l}< \\
0.02\end{array}$ & $\hat{0}_{0.04}$ & 0.02 \\
\hline W19 & 0.05 & $\begin{array}{l}<.2 \\
0.2\end{array}$ & 0.04 & 3.3 & $\begin{array}{l}<.01 \\
0.01\end{array}$ & 0.24 & 15.3 & 45 & $<1$ & 179.0 & 0.15 & $\hat{0}_{0.2}$ & 0.8 & $\dot{0}_{0.1}$ & 0.14 & $\begin{array}{l}<.02 \\
0.02\end{array}$ & $\hat{0}_{0.04}$ & 0.10 \\
\hline W20 & 0.05 & $\begin{array}{l}<.2 \\
0.2\end{array}$ & 0.08 & 4.8 & $\begin{array}{l}<.01 \\
0.01\end{array}$ & 0.31 & 5.4 & 51 & $<1$ & 145.0 & 0.05 & $\begin{array}{l}< \\
0.2\end{array}$ & 1.7 & $\dot{ }_{0.1}$ & 0.10 & $\begin{array}{l}<.02 \\
0.02\end{array}$ & $\begin{array}{l}<.04 \\
0.04\end{array}$ & 0.06 \\
\hline W21 & 0.05 & 0.3 & 0.05 & 4.2 & $\dot{0}_{0.01}$ & 0.23 & 4.9 & 15 & $<1$ & 50.0 & 0.12 & $\dot{0}_{0.2}$ & 0.7 & $\begin{array}{l}< \\
0.1\end{array}$ & 0.16 & $\begin{array}{l}<.02 \\
0.02\end{array}$ & $\hat{0}_{0.04}$ & 0.05 \\
\hline W22 & 0.04 & $\begin{array}{l}< \\
0.2\end{array}$ & 0.03 & 6.5 & $\begin{array}{l}< \\
0.01\end{array}$ & 0.46 & 53.2 & 68 & $<1$ & 338.0 & 0.13 & 0.4 & 1.2 & $\dot{0}_{0.1}$ & 0.33 & $\begin{array}{l}< \\
0.02\end{array}$ & $\dot{0}_{0.04}$ & 0.20 \\
\hline W23 & 0.04 & $\begin{array}{l}<.2 \\
0.2\end{array}$ & 0.03 & 4.0 & $\begin{array}{l}<.01 \\
0.01\end{array}$ & 0.29 & 8.4 & 76 & $<1$ & 349.0 & 0.09 & $\begin{array}{l}<.2 \\
0.2\end{array}$ & 1.7 & $\hat{0}_{0.1}$ & 0.24 & $\begin{array}{l}<.02 \\
0.02\end{array}$ & $\hat{0}_{0.04}$ & 0.12 \\
\hline W24 & 0.04 & 0.2 & 0.10 & 3.8 & $\begin{array}{l}< \\
0.01\end{array}$ & 0.37 & 13.5 & 41 & $<1$ & 318.0 & 0.05 & 0.3 & 1.2 & $\begin{array}{l}< \\
0.1\end{array}$ & 0.05 & 0.23 & $\hat{0}_{0.04}$ & 0.19 \\
\hline W25 & 0.04 & $\begin{array}{l}<.2 \\
0.2\end{array}$ & 0.03 & 3.4 & $\begin{array}{l}<.01 \\
0.01\end{array}$ & 0.16 & 5.1 & 20 & $<1$ & 28.0 & 0.06 & $\dot{0}_{0.2}$ & 1.6 & $\dot{ }_{0.1}$ & 0.07 & $\begin{array}{l}<.02 \\
0.02\end{array}$ & $\dot{0}_{0.04}$ & 0.07 \\
\hline W26 & 0.04 & 0.3 & 0.04 & 3.3 & $\begin{array}{l}<.01 \\
0.01\end{array}$ & 0.35 & 4.2 & 21 & $<1$ & 113.0 & 0.05 & $\dot{0}_{0.2}$ & 1.1 & $\dot{0}_{0.1}$ & 0.10 & $\begin{array}{l}<.02 \\
0.02\end{array}$ & $\begin{array}{l}<.04 \\
0.04\end{array}$ & 0.10 \\
\hline
\end{tabular}




\begin{tabular}{|c|c|c|c|c|c|c|c|c|c|c|c|c|c|c|c|c|c|c|}
\hline Analyte & Wgt & As & Cd & $\mathrm{Cu}$ & $\mathrm{Hg}$ & $\mathrm{Pb}$ & $\mathrm{Zn}$ & $\mathrm{Ba}$ & $\mathrm{Cr}$ & Mn & Mo & Co & $\mathrm{Ni}$ & Sn & Te & $\mathrm{Ag}$ & $\mathrm{Bi}$ & $\mathrm{Ce}$ \\
\hline W27 & 0.06 & $\hat{0}_{0.2}$ & 0.06 & 3.4 & $\begin{array}{l}< \\
0.01\end{array}$ & 0.41 & 9.0 & 94 & $<1$ & 359 & 0.05 & $\dot{0}_{0.2}$ & 1.4 & $\begin{array}{l}< \\
0.1\end{array}$ & 0.19 & $\begin{array}{l}< \\
0.02\end{array}$ & $\begin{array}{l}< \\
0.04\end{array}$ & 0.06 \\
\hline W28 & 0.04 & $\dot{0}_{0.2}$ & 0.03 & 1.5 & $\begin{array}{l}< \\
0.01\end{array}$ & 0.13 & 3.7 & 12 & $<1$ & 4.0 & 0.05 & $\begin{array}{l}< \\
0.2\end{array}$ & 0.4 & $\begin{array}{l}< \\
0.1\end{array}$ & 0.05 & $\begin{array}{l}<.02 \\
0.02\end{array}$ & $\begin{array}{l}< \\
0.04\end{array}$ & 0.03 \\
\hline W29 & 0.04 & $\begin{array}{l}< \\
0.2\end{array}$ & 0.06 & 3.9 & $\begin{array}{l}< \\
0.01\end{array}$ & 0.27 & 4.8 & 41 & $<1$ & 141.0 & 0.05 & $\begin{array}{l}< \\
0.2\end{array}$ & 1.9 & $\begin{array}{l}< \\
0.1\end{array}$ & 0.09 & 0.28 & $\begin{array}{l}< \\
0.04\end{array}$ & 0.11 \\
\hline W30 & 0.05 & $\begin{array}{l}< \\
0.2\end{array}$ & 0.13 & 3.4 & $\begin{array}{l}< \\
0.01\end{array}$ & 1.98 & 41.9 & 93 & $<1$ & 117.0 & 0.05 & 0.3 & 1.0 & $\begin{array}{l}< \\
0.1\end{array}$ & 0.38 & $\begin{array}{l}< \\
0.02\end{array}$ & $\begin{array}{l}< \\
0.04\end{array}$ & 0.22 \\
\hline W31 & 0.06 & $\begin{array}{l}< \\
0.2\end{array}$ & 0.03 & 8.9 & $\begin{array}{l}< \\
0.01\end{array}$ & 1.46 & 26.7 & 126 & $<1$ & 747.0 & 0.39 & 0.2 & 1.4 & $\begin{array}{l}< \\
0.1\end{array}$ & 0.24 & 0.21 & $\begin{array}{l}< \\
0.04\end{array}$ & 0.92 \\
\hline
\end{tabular}

A high strontium concentration of $127 \mathrm{ppm}$, which is 25.4 times the limit value ( $5 \mathrm{ppm}$ ) was found in the W9 sample. High and very high concentrations of strontium were determined in the W31 (175 ppm) and W1 samples (260 ppm, which translates into 52 times the limit value for solid fuels). The lowest strontium concentrations were observed in the W17 (8 ppm) and W24 samples (9 ppm). The highest concentrations of copper were recorded in the W22 (6.5 ppm), W8 (7.0 ppm), and W31 (8.9 ppm) samples, while the limit value was $2 \mathrm{ppm}$. On average, copper concentrations in the tested charcoals ranged from 2.4 $\mathrm{ppm}$ to $4.8 \mathrm{ppm}$. The concentration of zinc, ranging from $13.5 \mathrm{ppm}$ for the W24 sample to $50.2 \mathrm{ppm}$ for theW16 sample, exceeded the limit value of $10 \mathrm{ppm}$ in thirteen samples out of thirty-one. The lowest zinc concentration (3.5 ppm) was found in the W14 sample. The concentration of Ba ranged from 12 to 157 $\mathrm{ppm}$ and exceeded the limit value of $10 \mathrm{ppm}$ in all samples except for the W18 sample (4 ppm). The highest concentration of Ni was found in the W4 (4.8 $\mathrm{ppm})$ and W2 (5 ppm) samples, which is ten times the limit value $(0.5 \mathrm{ppm})$. Twenty-six samples exceeded the recommended values from 1 to 6 times; in the case of three samples, namely the W18 $(0.3 \mathrm{ppm})$, W28 $(0.4 \mathrm{ppm})$, and W11 $(0.5 \mathrm{ppm})$ samples, the Ni concentration is within the recommended values. The highest concentrations of manganese were found in samples W5 (717 ppm) and W31 (747 ppm), reaching a record value in the W10 sample (1817 ppm). In the three samples, namely W25, W1, and W14, the concentration of manganese ranges from 28 to 76 , well below the limit value of 83 ppm. Trace amounts of Mn, from 4 to 8 ppm, were observed in the W28 and W18 samples. In the case of four samples: W30, W17, W8, and W10, cadmium, with concentrations ranging from 0.13 to $0.23 \mathrm{ppm}$, exceeded the limit value of $0.1 \mathrm{ppm}$. The concentration of Cd was below $0.1 \mathrm{ppm}$ in the remaining 27 samples. In the W16 sample (0.5 ppm), a two and a half-fold increase above the recommended arsenic level $(0.2 \mathrm{ppm})$ was observed; in the case of next five samples, namely W7, W9, W10, W21, and W26 samples, the As concentration was 1.5 times the limit value $(0.2 \mathrm{ppm})$. In the remaining 25 samples, arsenic was found in trace amounts (below $0.2 \mathrm{ppm}$ ). When it comes to Se, slight exceedances of the limit values (by $0.1 \mathrm{ppm}$ ) were observed in samples W1, W23, W24, and W30. In the thirty samples, the mercury concentration was $<0.01 \mathrm{ppm}$. Hg concentrations reached the reference range of $0.02 \mathrm{ppm}$ only in the case of W4 sample. The remaining elements in the tested material, including $\mathrm{Ag}, \mathrm{Mo}, \mathrm{Cr}, \mathrm{Co}, \mathrm{Bi}, \mathrm{Ce}, \mathrm{Zn}$, and Te were present in trace amounts. The total content of toxic elements is presented in Tables 4 and 5. 
Table 4

Summary of the elemental content of the tested toxic charcoals with a very high and high degree of potential hazard to the environment and the health of living organisms.

\begin{tabular}{|c|c|c|c|c|c|c|c|c|c|c|c|c|}
\hline \multirow[t]{2}{*}{ Symbol } & As & Cd & $\mathrm{Cu}$ & $\mathrm{Hg}$ & $\mathrm{Pb}$ & $\mathrm{Zn}$ & $\Sigma$ & $\mathrm{Ba}$ & $\mathrm{Cr}$ & Mn & Mo & $\Sigma$ \\
\hline & ppm & ppm & ppm & ppm & ppm & ppm & ppm & ppm & ppm & ppm & ppm & ppm \\
\hline W1 & $<0.2$ & 0.02 & 4.5 & $<0.01$ & 1.58 & 7.6 & 13.91 & 98 & 1 & 32.0 & 1.24 & 132.24 \\
\hline W2 & $<0.2$ & 0.02 & 3.8 & $<0.01$ & 0.36 & 7.7 & 12.09 & 96 & $<1$ & 124.0 & 0.24 & 221.24 \\
\hline W3 & $<0.2$ & 0.02 & 4.0 & $<0.01$ & 0.26 & 6.0 & 26 & 157 & $<1$ & 240.0 & 0.10 & 353.48 \\
\hline W4 & 0.2 & 0.09 & 4.1 & 0.02 & 0.92 & 31.8 & 37.13 & 125 & 1 & 218.0 & 0.08 & 344.08 \\
\hline W5 & $<0.2$ & 0.03 & 5.8 & $<0.01$ & 0.27 & 6.6 & 12.91 & 57 & $<1$ & 717.0 & 0.09 & 775.09 \\
\hline W6 & $<0.2$ & 0.07 & 4.2 & $<0.01$ & 0.81 & 26.0 & 50.04 & 109 & 1 & 231.0 & 0.08 & 1119.17 \\
\hline W7 & 0.3 & 0.05 & 3.7 & $<0.01$ & 0.38 & 8.4 & 12.84 & 125 & $<1$ & 273.0 & 0.06 & 399.06 \\
\hline W8 & $<0.2$ & 0.20 & 7.0 & $<0.01$ & 1.50 & 47.3 & 56.21 & 44 & $<1$ & 127.0 & 0.07 & 172.07 \\
\hline W9 & 0.3 & 0.09 & 4.0 & $<0.01$ & 0.69 & 8.0 & 69.05 & 129 & $<1$ & 220.0 & 0.81 & 571.13 \\
\hline W10 & 0.3 & 0.23 & 3.4 & $<0.01$ & 1.98 & 41.9 & 47.82 & 97 & $<1$ & 1817.0 & 0.05 & 1915.05 \\
\hline W11 & $<0.2$ & 0.03 & 4.0 & $<0.01$ & 0.36 & 4.5 & 9.1 & 44 & $<1$ & 127.0 & 0.05 & 172.05 \\
\hline W12 & 0.2 & 0.09 & 3.5 & $<0.01$ & 1.65 & 23.1 & 56.92 & 47 & $<1$ & 366.0 & 0.09 & 2087.1 \\
\hline W13 & 0.2 & 0.09 & 2.4 & $<0.01$ & 2.0 & 11.3 & 16 & 63 & $<1$ & 183.0 & 0.09 & 247.09 \\
\hline W14 & $<0.2$ & 0.04 & 5.1 & $<0.01$ & 0.23 & 3.5 & 9.08 & 37 & $<1$ & 76.0 & 0.05 & 114.05 \\
\hline W15 & $<0.2$ & 0.04 & 3.7 & $<0.01$ & 0.36 & 9.0 & 25.08 & 46 & $<1$ & 272.0 & 0.07 & 361.14 \\
\hline W16 & 0.5 & 0.02 & 4.5 & $<0.01$ & 0.65 & 60.2 & 65.88 & 79 & $<1$ & 293.0 & 0.05 & 373.05 \\
\hline W17 & $<0.2$ & 0.15 & 2.9 & $<0.01$ & 0.97 & 21.6 & 25.83 & 71 & $<1$ & 169.0 & 0.09 & 241.09 \\
\hline W18 & $<0.2$ & 0.02 & 2.7 & $<0.01$ & 0.19 & 9.6 & 91.71 & 4 & $<1$ & 8.0 & 0.05 & 614.14 \\
\hline W19 & $<0.2$ & 0.04 & 3.3 & $<0.01$ & 0.24 & 15.3 & 19.09 & 45 & $<1$ & 179.0 & 0.15 & 225.15 \\
\hline W20 & $<0.2$ & 0.08 & 4.8 & $<0.01$ & 0.31 & 5.4 & 10.8 & 51 & $<1$ & 145.0 & 0.05 & 197.05 \\
\hline W21 & 0.3 & 0.05 & 4.2 & $<0.01$ & 0.23 & 4.9 & 29.89 & 15 & $<1$ & 50.0 & 0.12 & 422.2 \\
\hline W22 & $<0.2$ & 0.03 & 6.5 & $<0.01$ & 0.46 & 53.2 & 60.4 & 68 & $<1$ & 338.0 & 0.13 & 407.13 \\
\hline W23 & $<0.2$ & 0.03 & 4.0 & $<0.01$ & 0.29 & 8.4 & 12.93 & 76 & $<1$ & 349.0 & 0.09 & 426.09 \\
\hline W24 & 0.2 & 0.10 & 3.8 & $<0.01$ & 0.37 & 13.5 & 73.33 & 41 & $<1$ & 318.0 & 0.05 & 833.22 \\
\hline W25 & $<0.2$ & 0.03 & 3.4 & $<0.01$ & 0.16 & 5.1 & 8.9 & 20 & $<1$ & 28.0 & 0.06 & 49.06 \\
\hline W26 & 0.3 & 0.04 & 3.3 & $<0.01$ & 0.35 & 4.2 & 8.2 & 21 & $<1$ & 113.0 & 0.05 & 135.05 \\
\hline W27 & $<0.2$ & 0.06 & 3.4 & $<0.01$ & 0.41 & 9.0 & 17.1 & 94 & $<1$ & 359 & 0.05 & 184.11 \\
\hline W28 & $<0.2$ & 0.03 & 1.5 & $<0.01$ & 0.13 & 3.7 & 5.57 & 12 & $<1$ & 4.0 & 0.05 & 17.05 \\
\hline W29 & $<0.2$ & 0.06 & 3.9 & $<0.01$ & 0.27 & 4.8 & 9.24 & 41 & $<1$ & 141.0 & 0.05 & 183.05 \\
\hline W30 & $<0.2$ & 0.13 & 3.4 & $<0.01$ & 1.98 & 41.9 & 14.81 & 93 & $<1$ & 117.0 & 0.05 & 200.1 \\
\hline W31 & $<0.2$ & 0.03 & 8.9 & $<0.01$ & 1.46 & 26.7 & 37.3 & 126 & $<1$ & 747.0 & 0.39 & 874.39 \\
\hline
\end{tabular}


Table 5

Summary of the elemental content of the tested toxic elements representing a medium degree of hazard to the environment and the health of living organisms.

\begin{tabular}{|c|c|c|c|c|c|c|c|c|c|c|c|c|}
\hline \multirow[t]{2}{*}{ symbol } & Co & $\mathrm{Ni}$ & Sn & $\mathrm{Te}$ & $\Sigma$ & $\mathrm{Ag}$ & $\mathrm{Bi}$ & $\mathrm{Ce}$ & $\mathrm{Se}$ & $\mathrm{Sr}$ & $\mathrm{Zr}$ & $\Sigma$ \\
\hline & ppm & ppm & ppm & ppm & ppm & ppm & ppm & ppm & ppm & ppm & ppm & ppm \\
\hline W1 & 0.3 & 0.9 & $<0.1$ & 0.88 & 2.18 & $<0.2$ & $<0.04$ & 1.73 & 0.4 & 260 & 0.3 & 48.83 \\
\hline W2 & 0.2 & 5.0 & $<0.1$ & 0.20 & 5.5 & $<0.2$ & $<0.04$ & 0.29 & $<0.3$ & 47 & 1.0 & 311.5 \\
\hline W3 & 0.2 & 2.0 & $<0.1$ & 0.16 & 7.68 & $<0.2$ & $<0.04$ & 0.10 & $<0.3$ & 25 & 0.4 & 92.2 \\
\hline W4 & 0.2 & 4.8 & $<0.1$ & 0.30 & 5.4 & 0.2 & $<0.04$ & 0.06 & 0.3 & 91 & 0.6 & 18.91 \\
\hline W5 & 0.2 & 3.1 & $<0.1$ & 0.09 & 3.49 & 0.24 & $<0.04$ & 0.13 & $<0.3$ & 18 & $<0.2$ & 111.11 \\
\hline W6 & 0.2 & 4.6 & $<0.1$ & 0.18 & 8.89 & $<0.2$ & $<0.04$ & 0.09 & $<0.3$ & 83 & 0.4 & 32.95 \\
\hline W7 & 0.2 & 1.8 & $<0.1$ & 0.13 & 2.23 & 0.33 & $<0.04$ & 0.08 & $<0.3$ & 32 & $<0.2$ & 18.72 \\
\hline W8 & $<0.2$ & 0.6 & $<0.1$ & 0.05 & 0.95 & 0.82 & $<0.04$ & 0.06 & $<0.3$ & 17 & 0.5 & 51.67 \\
\hline W9 & $<0.2$ & 1.0 & $<0.1$ & 0.58 & 3.18 & $<0.2$ & $<0.04$ & 0.59 & $<0.3$ & 127 & 0.6 & 27.92 \\
\hline W10 & 0.5 & 1.0 & $<0.1$ & 0.11 & 1.71 & $<0.2$ & $<0.04$ & 0.08 & $<0.3$ & 27 & 0.3 & 10.77 \\
\hline W11 & $<0.2$ & 0.5 & $<0.1$ & 0.12 & 0.92 & $<0.2$ & $<0.04$ & 0.03 & $<0.3$ & 10 & $<0.2$ & 38.69 \\
\hline W12 & $<0.2$ & 1.0 & $<0.1$ & 0.18 & 2.63 & 0.34 & $<0.04$ & 0.18 & $<0.3$ & 16 & 0.2 & 47.83 \\
\hline W13 & 0.2 & 0.9 & $<0.1$ & 0.29 & 1.49 & $<0.2$ & $<0.04$ & 0.09 & $<0.3$ & 47 & $<0.2$ & 34.83 \\
\hline W14 & $<0.2$ & 1.5 & $<0.1$ & 0.20 & 2 & $<0.2$ & $<0.04$ & 0.09 & $<0.3$ & 34 & 0.2 & 82.66 \\
\hline W15 & 0.4 & 2.4 & $<0.1$ & 0.14 & 3.49 & 0.21 & $<0.04$ & 0.17 & $<0.3$ & 44 & $<0.2$ & 25.85 \\
\hline W16 & 0.2 & 1.5 & $<0.1$ & 0.12 & 1.92 & $<0.2$ & $<0.04$ & 0.11 & $<0.3$ & 25 & $<0.2$ & 9.77 \\
\hline W17 & $<0.2$ & 1.5 & $<0.1$ & 0.16 & 1.96 & $<0.2$ & $<0.04$ & 0.93 & 0.3 & 8 & 0.3 & 35.62 \\
\hline W18 & $<0.2$ & 0.3 & $<0.1$ & 0.10 & 3.88 & $<0.2$ & $<0.04$ & 0.02 & $<0.3$ & 53 & 0.2 & 38.84 \\
\hline W19 & $<0.2$ & 0.8 & $<0.1$ & 0.14 & 1.24 & $<0.2$ & $<0.04$ & 0.10 & $<0.3$ & 38 & $<0.2$ & 14.8 \\
\hline W20 & $<0.2$ & 1.7 & $<0.1$ & 0.10 & 2.1 & $<0.2$ & $<0.04$ & 0.06 & $<0.3$ & 14 & 0.2 & 53.64 \\
\hline W21 & $<0.2$ & 0.7 & $<0.1$ & 0.16 & 3.34 & $<0.2$ & $<0.04$ & 0.05 & $<0.3$ & 65 & $<0.2$ & 54.94 \\
\hline W22 & 0.4 & 1.2 & $<0.1$ & 0.33 & 2.03 & $<0.2$ & $<0.04$ & 0.20 & $<0.3$ & 54 & $<0.2$ & 37.96 \\
\hline W23 & $<0.2$ & 1.7 & $<0.1$ & 0.24 & 2.24 & $<0.2$ & $<0.04$ & 0.12 & 0.4 & 37 & $<0.2$ & 92.9 \\
\hline W24 & 0.3 & 1.2 & $<0.1$ & 0.05 & 4.27 & 0.23 & $<0.04$ & 0.19 & 0.4 & 9 & 0.3 & 14.81 \\
\hline W25 & $<0.2$ & 1.6 & $<0.1$ & 0.07 & 1.97 & $<0.2$ & $<0.04$ & 0.07 & $<0.3$ & 14 & 0.2 & 16.84 \\
\hline W26 & $<0.2$ & 1.1 & $<0.1$ & 0.10 & 1.5 & $<0.2$ & $<0.04$ & 0.10 & $<0.3$ & 16 & $<0.2$ & 31.65 \\
\hline W27 & $<0.2$ & 1.4 & $<0.1$ & 0.19 & 3.47 & $<0.2$ & $<0.04$ & 0.06 & 0.3 & 25 & $<0.2$ & 16.77 \\
\hline W28 & $<0.2$ & 0.4 & $<0.1$ & 0.05 & 0.75 & $<0.2$ & $<0.04$ & 0.03 & $<0.3$ & 16 & $<0.2$ & 17.93 \\
\hline W29 & $<0.2$ & 1.9 & $<0.1$ & 0.09 & 2.29 & 0.28 & $<0.04$ & 0.11 & $<0.3$ & 17 & $<0.2$ & 34.7 \\
\hline W30 & 0.3 & 1.0 & $<0.1$ & 0.38 & 3.04 & $<0.2$ & $<0.04$ & 0.22 & 0.4 & 27 & 0.3 & 176.87 \\
\hline W31 & 0.2 & 1.4 & $<0.1$ & 0.24 & 1.94 & 0.21 & $<0.04$ & 0.92 & 0.3 & 175 & 0.4 & 262.67 \\
\hline
\end{tabular}

The extract yields of the charcoals investigated varied in the range of $0.06-0.35 \%$ wt. The highest extract yields were found for the W14 (0.35\% wt.) sample from the gravity method and the lowest for the W4 sample of unknown production method (Table 2).

The investigated charcoals extracts were very rich in polycyclic aromatic hydrocarbons, both in the respect of PAHs concentrations and the number of compounds occurring. The following compounds were identified: naphthalene $(\mathrm{N})(\mathrm{m} / \mathrm{z}=128)$, biphenyl $(B)(\mathrm{m} / \mathrm{z}=154)$, acenaphthene $(A c)(\mathrm{m} / \mathrm{z}=154)$, acenaphthylene (Ace) $(\mathrm{m} / \mathrm{z}=152)$, fluorene $(F)(\mathrm{m} / \mathrm{z}=166)$, phenanthrene $(P)(\mathrm{m} / \mathrm{z}=178)$, anthracene $(A)(\mathrm{m} / \mathrm{z}=178)$, methylenophenanthrene $($ MeP) $(\mathrm{m} / \mathrm{z}=$ 190), fluoranthene $(F I)(m / z=202)$, acenthrylene (Acen) $(\mathrm{m} / \mathrm{z}=202)$, acephenanthrylene (AceP) $(\mathrm{m} / \mathrm{z}=202)$, pyrene (Py) ( $\mathrm{m} / \mathrm{z}=202)$, benzo(c)phenanthrene $(B(c) P)(m / z=228)$, benzo(a)anthracene $(B(a) A)(m / z=228)$, chrysene $(C h)(m / z=228)$, benzo(ghi)fluoranthene $(B(g h i) F I)(m / z=226)$, acepyrene $(A c e P y)$ $(\mathrm{m} / \mathrm{z}=226)$, benzofluoranthenes (BF) $(\mathrm{m} / \mathrm{z}=252)$, benzopyrenes (BP) $(\mathrm{m} / \mathrm{z}=252)$, perylene (Per) $(\mathrm{m} / \mathrm{z}=252)$, indeno [cd-1,2,3]pyrene $(I P)(\mathrm{m} / \mathrm{z}=276)$, and benzo(ghi)perylene (B(ghi)Per) ( $\mathrm{m} / \mathrm{z}=276)$. The generalized PAHs distribution is shown in the Supplement (Fig. 1). Most of unsubstituted PAHs were accompanied by their alkyl C1-C3 derivatives. Phenalenes and PAHs heavier than 6-ring compounds were not found in the charcoal extracts. The most 
numerous unsubstituted PAHs were in the W14 sample (25 identified compounds and the least numerous in the W11, W17 and W28 samples (11 identified compounds).

Generally, the highest concentrations in distribution were shown by the 5-ring PAHs, benzo(b) - and benzo(k)fluoranthenes, 91.70 and 31.21 ng/g (aver.) respectively, that were followed by benzo(a)pyrenes and benzo(e)pyrenes, 72.45 and $69.63 \mathrm{ng} / \mathrm{g}$ (aver.), respectively, and perylene, $14.98 \mathrm{ng} / \mathrm{g}$ (aver.). Lighter 2-3-ring PAHs were the minor charcoal extracts components, most probably because they evaporated during wood pyrolysis (Fig. 2).

Only naphthalene showed elevated contents in some of the samples, up to $50.20 \mathrm{ng} / \mathrm{g}$ (W6, W8, W10, and W13). Anthracene, considered to be a product of biomass/coal combustion ${ }^{14}$, showed relatively low concentrations, $0.03-5.43 \mathrm{ng} / \mathrm{g}, 0.94 \mathrm{ng} / \mathrm{g}$ on aver.) comparing to phenanthrene, $0.13-32.46 \mathrm{ng} / \mathrm{g}, 5.44$ $\mathrm{ng} / \mathrm{g}$ on aver. (Table 6). This is reflected by P/A ratio being in the range from 2.98 (W17) to 13.95 (W6) (Table 7).

Table 6

Polycyclic aromatic hydrocarbons concentrations in charcoals investigated [pp

\begin{tabular}{|c|c|c|c|c|c|c|c|c|c|c|c|c|c|c|c|c|c|}
\hline Sample & $\mathbf{N}$ & $\mathrm{Bi}$ & Ace & Acy & $\mathbf{F}$ & $P$ & A & MeP & FI & Acen & AcPhe & Py & BcPhe & $\mathrm{BaA}$ & $\mathrm{Ch}$ & BghiFI & AcPy \\
\hline W1 & 0.16 & 4.84 & 0.58 & - & 0.34 & 4.34 & 3.93 & - & 1.39 & 0.07 & 0.14 & 0.80 & 0.07 & 0.18 & 0.15 & 0.04 & 0.10 \\
\hline W2 & 0.04 & 1.55 & - & - & 0.23 & 0.71 & 0.07 & - & 0.40 & 0.01 & 0.05 & 0.20 & 0.02 & 0.11 & 0.02 & - & - \\
\hline W3 & 0.40 & 0.13 & - & - & 0.13 & 0.65 & 0.10 & - & 0.40 & - & - & 0.30 & - & - & - & - & - \\
\hline W4 & 0.09 & - & - & - & - & 0.53 & 0.06 & - & 0.25 & 0.02 & 0.04 & 0.20 & 0.01 & 0.11 & 0.01 & 0.03 & 0.05 \\
\hline W5 & 0.47 & 8.93 & 0.15 & 0.85 & 0.20 & 1.25 & 0.16 & 0.19 & 1.00 & 0.06 & 0.09 & 0.63 & 0.06 & 0.20 & 0.03 & 0.03 & 0.03 \\
\hline W6 & 29.21 & 12.08 & 0.12 & - & - & 20.89 & 1.50 & - & 36.89 & 1.45 & 2.38 & 26.33 & 1.30 & 4.27 & 2.38 & 2.65 & 1.34 \\
\hline W7 & 1.43 & 1.56 & 0.64 & 0.92 & - & 5.45 & 0.70 & - & 4.66 & 0.49 & 0.54 & 3.37 & 0.27 & 1.35 & 0.65 & 0.30 & - \\
\hline W8 & 21.96 & 6.56 & 0.28 & - & 1.51 & 19.09 & 3.05 & 5.13 & 16.84 & 0.62 & 0.67 & 11.96 & 0.66 & 4.23 & 1.41 & 0.85 & 0.16 \\
\hline W9 & 0.19 & 0.67 & 0.04 & 0.07 & - & 5.00 & 0.99 & - & 2.13 & 0.09 & 0.17 & 1.77 & 0.10 & 0.52 & 0.28 & 0.09 & 0.13 \\
\hline W10 & 12.45 & 2.53 & 0.05 & 1.40 & - & 2.49 & 0.30 & - & 3.22 & - & 0.17 & 1.65 & 0.15 & 0.23 & 0.09 & 0.18 & 0.06 \\
\hline W11 & - & - & - & - & - & 0.13 & 0.03 & - & 0.23 & - & 0.01 & 0.06 & - & - & - & - & - \\
\hline W12 & 6.78 & 3.09 & 0.19 & 2.17 & 0.18 & 7.01 & 0.94 & 0.86 & 5.51 & 0.22 & 0.38 & 3.59 & 0.25 & 1.03 & 0.50 & 0.38 & 0.27 \\
\hline W13 & 1.79 & 1.59 & - & - & 0.14 & 3.00 & 0.44 & 0.15 & 2.49 & 0.17 & 0.21 & 1.97 & 0.21 & 1.10 & 0.58 & 0.12 & 0.10 \\
\hline W14 & 7.92 & 3.34 & 0.28 & 4.01 & 1.91 & 11.00 & 1.47 & 2.23 & 12.48 & 0.47 & 0.87 & 7.95 & 0.45 & 1.88 & 0.72 & 0.68 & 0.25 \\
\hline W15 & 50.20 & 9.29 & 0.14 & 0.74 & 0.05 & 32.46 & 5.43 & 3.44 & 19.79 & 0.82 & 0.92 & 16.63 & 2.03 & 17.67 & 6.04 & 0.75 & - \\
\hline W16 & - & - & - & - & - & - & - & - & - & - & - & - & - & - & - & - & - \\
\hline W17 & - & 0.20 & - & - & - & 0.23 & 0.08 & - & 0.23 & - & 0.03 & 0.14 & - & - & - & - & - \\
\hline W18 & - & 0.22 & - & - & - & 0.71 & 0.19 & - & 24.95 & - & 0.05 & 0.36 & 0.07 & 0.15 & 0.09 & - & - \\
\hline W19 & - & 0.41 & 0.08 & - & - & 0.59 & 0.11 & - & 0.54 & - & 0.04 & 0.50 & 0.16 & 0.46 & 0.13 & 0.26 & 0.07 \\
\hline W20 & - & - & - & - & - & - & - & - & - & - & - & - & - & - & - & - & - \\
\hline W21 & 0.85 & - & - & - & 0.20 & 4.93 & 1.21 & 0.83 & 4.27 & - & - & 3.17 & 0.31 & 1.87 & 0.69 & 0.24 & - \\
\hline W22 & 0.86 & 1.52 & - & - & 0.14 & 0.60 & 0.10 & & 0.45 & - & - & 0.36 & 0.08 & 0.56 & 0.09 & - & - \\
\hline W23 & & & - & - & & 0.72 & 0.14 & & 0.48 & - & - & 0.30 & 0.03 & 0.09 & 0.02 & - & - \\
\hline W24 & - & - & - & - & - & - & - & - & - & - & - & - & - & - & - & - & - \\
\hline W25 & - & - & - & - & - & - & - & - & - & - & - & - & - & - & - & - & - \\
\hline W26 & - & - & 9.37 & - & 0.13 & 6.35 & 1.09 & 0.55 & 9.26 & - & 0.31 & 5.68 & - & - & - & 0.45 & - \\
\hline W27 & - & - & - & - & - & - & - & - & - & - & - & - & - & - & - & - & - \\
\hline W28 & - & - & 0.06 & - & - & 0.52 & 0.13 & - & 0.77 & - & - & 0.46 & - & - & - & - & - \\
\hline W29 & - & - & - & - & - & - & - & - & - & - & - & - & - & - & - & - & - \\
\hline W30 & & & 0.44 & - & 0.22 & 1.80 & 0.31 & - & 1.80 & - & - & 1.26 & - & - & - & 0.12 & - \\
\hline W31 & - & - & - & - & - & - & - & - & - & - & - & - & - & - & - & - & - \\
\hline
\end{tabular}

$\mathrm{N}$ - naphthalene, $\mathrm{Bi}$ - biphenyl, Ace - acenaphene, Acy - acenaphthylene, $\mathrm{F}$ - fluorene, $\mathrm{P}$ - phenanthrene, $\mathrm{A}$ - anthracene, MeP - methylenephenanthrene, $\mathrm{FI}$ fluoranthene, Acen - acenthrylene, AcPhe - acephenanthrylene, Py - pyrene, BcPhe - benzo(c)phenanthrene, BaA - 
benzo(a)anthracene, Ch - chrysene, BghiFI - benzo(ghi)fluoranthene, AcPy - acepyrene, BbF - benzo(b)fluoranthene, BkF - benzo(k)fluoranthene, BaF benzo(a)fluoranthene, BaP - benzo(a)pyrene, BeP - benzo(e)pyrene, Pe - perylene, IP - indeno[1,2,3-cd]pyrene, BghiP - benzo(ghi)perylene

Table 7

Values of diagnostic PAHs ratios for the charcoals investigated

\begin{tabular}{|c|c|c|c|c|c|c|c|c|c|c|c|}
\hline Sample & P/A & $A /(A+P)$ & $\mathrm{Fl} /(\mathrm{Fl}+\mathrm{Py})$ & $\mathrm{Fl} / \mathrm{Py}$ & $\mathrm{Fl} /(\mathrm{FI}+\mathrm{P})$ & $\begin{array}{l}\mathrm{BaA} \\
(\mathrm{BaA}+\mathrm{Ch})\end{array}$ & BaP/ BghiP & IP/ BghiP & IP/ (IP + BghiP) & $\mathrm{BaA} / \mathrm{BaP}$ & $\mathrm{Py} / \mathrm{BaP}$ \\
\hline W1 & 1.10 & 0.48 & 0.64 & 1.74 & 0.24 & 0.55 & 52.52 & 3.05 & 0.75 & 0.02 & 0.09 \\
\hline W2 & 10.48 & 0.09 & 0.67 & 2.04 & 0.36 & 0.86 & - & - & - & 0.01 & 0.02 \\
\hline W3 & 6.17 & 0.14 & 0.58 & 1.36 & 0.38 & - & - & - & - & 0.00 & 0.14 \\
\hline W4 & 9.01 & 0.10 & 0.56 & 1.25 & 0.32 & 0.95 & - & - & - & 0.02 & 0.04 \\
\hline W5 & 7.96 & 0.11 & 0.61 & 1.58 & 0.44 & 0.89 & - & - & - & 0.06 & 0.19 \\
\hline W6 & 13.95 & 0.07 & 0.58 & 1.40 & 0.64 & 0.64 & 54.04 & 10.03 & 0.91 & 0.03 & 0.16 \\
\hline W7 & 7.83 & 0.11 & 0.58 & 1.38 & 0.46 & 0.68 & 61.77 & 7.55 & 0.88 & 0.03 & 0.07 \\
\hline W8 & 6.26 & 0.14 & 0.58 & 1.41 & 0.47 & 0.75 & 62.36 & 5.80 & 0.85 & 0.04 & 0.11 \\
\hline W9 & 5.06 & 0.16 & 0.55 & 1.21 & 0.30 & 0.65 & - & - & - & 0.02 & 0.08 \\
\hline W10 & 8.38 & 0.11 & 0.66 & 1.95 & 0.56 & 0.73 & 130.97 & 6.47 & 0.87 & 0.01 & 0.07 \\
\hline W11 & 4.71 & 0.18 & 0.78 & 3.61 & 0.64 & - & - & - & - & 0.00 & 0.01 \\
\hline W12 & 7.44 & 0.12 & 0.61 & 1.54 & 0.44 & 0.67 & 115.20 & 10.81 & 0.92 & 0.03 & 0.10 \\
\hline W13 & 6.79 & 0.13 & 0.56 & 1.26 & 0.45 & 0.66 & 54.28 & 10.82 & 0.92 & 0.03 & 0.05 \\
\hline W14 & 7.46 & 0.12 & 0.61 & 1.57 & 0.53 & 0.72 & 74.01 & 8.47 & 0.89 & 0.03 & 0.11 \\
\hline W15 & 5.98 & 0.14 & 0.54 & 1.19 & 0.38 & 0.75 & 172.93 & 4.52 & 0.82 & 0.02 & 0.02 \\
\hline W16 & - & - & - & - & - & - & - & - & - & - & - \\
\hline W17 & 2.89 & 0.26 & 0.63 & 1.70 & 0.50 & - & - & - & - & 0.00 & 0.07 \\
\hline W18 & 3.78 & 0.21 & 0.99 & 69.87 & 0.97 & 0.64 & - & - & - & 0.00 & 0.01 \\
\hline W19 & 5.60 & 0.15 & 0.52 & 1.08 & 0.48 & 0.78 & - & - & 1.00 & 0.02 & 0.02 \\
\hline W20 & - & - & - & - & - & - & - & - & - & - & - \\
\hline W21 & 4.08 & 0.20 & 0.57 & 1.35 & 0.46 & 0.73 & 955.55 & 13.31 & 0.93 & 0.03 & 0.05 \\
\hline W22 & 6.15 & 0.14 & 0.56 & 1.25 & 0.43 & 0.86 & 49.82 & 4.49 & 0.82 & 0.04 & 0.02 \\
\hline W23 & 5.16 & 0.16 & 0.61 & 1.58 & 0.40 & 0.79 & - & - & - & 0.01 & 0.05 \\
\hline W24 & - & - & - & - & - & - & - & - & - & - & - \\
\hline W25 & - & - & - & - & - & - & - & - & - & - & - \\
\hline W26 & 5.80 & 0.15 & 0.62 & 1.63 & 0.59 & - & - & - & - & 0.00 & 0.06 \\
\hline W27 & - & - & - & - & - & - & - & - & - & - & - \\
\hline W28 & 3.95 & 0.20 & 0.62 & 1.66 & 0.59 & - & - & - & - & 0.00 & 0.06 \\
\hline W29 & - & - & - & - & - & - & - & - & - & - & - \\
\hline W30 & 5.91 & 0.14 & 0.59 & 1.43 & 0.50 & - & - & - & - & 0.00 & 0.04 \\
\hline W31 & - & - & - & - & - & - & - & - & - & - & - \\
\hline
\end{tabular}

$\mathrm{P}$ - phenanthrene, A - anthracene, FI - fluoranthene, Py - pyrene, BaA - benzo(a)anthracene, Ch - chrysene, BaP - benzo(a)pyrene, BeP - benzo(e)pyrene, Pe perylene, IP - indeno[1,2,3-cd]pyrene, BghiP - benzo(ghi)perylene

The four types of PAHs distribution can be distinguished within the sample set as shown in Fig. 3, (i) with the increased content of lighter 2-4 ring PAHs, with the increased content of lighter 2-4 ring PAHs, Light/Heavy PAHs ratio 0.15-0.31 (samples W1, W3, W5, W9, W12, and W18), (ii) with very low content of lighter PAHs, Light/Heavy PAHs ratio 0.01-0.06 (W11, W13, 15, W17, W19, W21, and W22), (iii) with benzofluoranthenes (Bf) dominating over benzopyrenes $(\mathrm{Bp})$, with $\sum \mathrm{Bf} / \sum \mathrm{Bp}$ ratio $>1.0\left(\mathrm{~W} 1, \mathrm{~W} 3, \mathrm{~W} 5, \mathrm{~W} 6, \mathrm{~W} 8\right.$, and $\mathrm{W} 17$ samples and (iiii) benzopyrenes dominating over benzofluoranthenes, with $\sum \mathrm{Bf} / \sum \mathrm{Bp}$ ratio > 1.0 (the rest of the sample set) (Table 8 ). 
Table 8

Indices assessing possible health and environmental impact of charcoal investigated

\begin{tabular}{|c|c|c|c|c|c|c|c|c|}
\hline Sample & $\begin{array}{l}\sum \mathrm{Bf} / \sum \mathrm{Bp} \\
(1)\end{array}$ & PAH sum (2) & $\begin{array}{l}\text { TEQ } \\
\text { (3) }\end{array}$ & $\begin{array}{l}\sum \mathrm{PAH}_{\text {card }} \\
\sum \mathrm{PAH}_{\text {tot }} \\
\text { (4) }\end{array}$ & $\begin{array}{l}\text { BaPE } \\
(5)\end{array}$ & $\begin{array}{l}\mathrm{BaPE} / \mathrm{BaP} \\
(6)\end{array}$ & $\begin{array}{l}\text { MEQ } \\
(7)\end{array}$ & $\begin{array}{l}\mathrm{L} / \mathrm{H} \\
(8)\end{array}$ \\
\hline W1 & 1.53 & 53.37 & 10.79 & 0.59 & 10.35 & 1.14 & 10.24 & 0.45 \\
\hline W2 & 0.52 & 21.61 & 8.59 & 0.87 & 8.41 & 1.05 & 8.52 & 0.18 \\
\hline W3 & 1.76 & 12.55 & 2.73 & 0.83 & 2.53 & 1.22 & 2.63 & 0.20 \\
\hline W4 & 0.66 & 15.79 & 5.04 & 0.77 & 4.89 & 1.08 & 4.83 & 0.08 \\
\hline W5 & 1.55 & 38.03 & 4.57 & 0.68 & 4.20 & 1.25 & 4.32 & 0.58 \\
\hline W6 & 1.39 & 1156.47 & 224.43 & 0.82 & 208.78 & 1.26 & 202.78 & 0.13 \\
\hline W7 & 0.97 & 212.81 & 60.77 & 0.82 & 58.45 & 1.14 & 57.57 & 0.10 \\
\hline W8 & 1.14 & 639.88 & 136.91 & 0.78 & 129.56 & 1.18 & 125.90 & 0.16 \\
\hline W9 & 0.63 & 80.08 & 24.48 & 0.81 & 23.71 & 1.08 & 23.55 & 0.16 \\
\hline W10 & 0.79 & 111.34 & 27.21 & 0.71 & 26.23 & 1.11 & 26.57 & 0.28 \\
\hline W11 & 0.57 & 15.06 & 5.51 & 0.84 & 5.37 & 1.06 & 5.38 & 0.03 \\
\hline W12 & 0.83 & 163.71 & 42.76 & 0.76 & 41.15 & 1.12 & 39.59 & 0.23 \\
\hline W13 & 0.82 & 170.40 & 45.77 & 0.82 & 44.06 & 1.14 & 43.32 & 0.08 \\
\hline W14 & 0.91 & 388.91 & 88.79 & 0.77 & 84.79 & 1.15 & 81.79 & 0.16 \\
\hline W15 & 0.67 & 3554.11 & 1023.87 & 0.90 & 987.11 & 1.11 & 984.98 & 0.04 \\
\hline W16 & - & - & - & - & - & - & - & - \\
\hline W17 & 1.77 & 12.55 & 2.77 & 0.94 & 2.54 & 1.25 & 2.60 & 0.08 \\
\hline W18 & 0.85 & 192.50 & 42.83 & 0.83 & 40.62 & 1.14 & 42.62 & 0.16 \\
\hline W19 & 0.44 & 131.15 & 31.61 & 0.86 & 30.42 & 1.11 & 31.76 & 0.02 \\
\hline W20 & - & - & - & - & - & - & - & - \\
\hline W21 & 0.82 & 258.47 & 74.27 & 0.86 & 71.20 & 1.11 & 74.02 & 0.06 \\
\hline W22 & 0.33 & 73.34 & 16.63 & 0.81 & 16.30 & 1.09 & 16.48 & 0.06 \\
\hline W23 & 0.96 & 20.68 & 7.12 & 0.78 & 6.87 & 1.09 & 6.32 & 0.09 \\
\hline W24 & - & - & - & - & - & - & - & - \\
\hline W25 & - & - & - & - & - & - & - & - \\
\hline W26 & 0.82 & 442.16 & 116.49 & 0.87 & 111.29 & 1.12 & 118.86 & 0.08 \\
\hline W27 & - & - & - & - & - & - & - & - \\
\hline W28 & 0.76 & 28.31 & 8.21 & 0.75 & 7.94 & 1.09 & 8.62 & 0.07 \\
\hline W29 & - & - & - & - & - & - & - & - \\
\hline W30 & 0.49 & 88.79 & 37.65 & 0.82 & 36.94 & 1.05 & 37.48 & 0.07 \\
\hline W31 & - & - & - & - & - & - & - & - \\
\hline
\end{tabular}

Several PAHs diagnostic ratios were calculated basing on the quantitative analysis15-16 (Table 7). On the PAHs diagnostic diagrams of Fl/(FI+ Py) ratio over $\mathrm{A} /(\mathrm{A}+\mathrm{P}), \mathrm{BaA} /(\mathrm{Ch}+\mathrm{BaA})$, and $\mathrm{P} /(\mathrm{IP}+\mathrm{BghiPer})$ ratios (Fig. 4) all samples group closely each other in the area corresponding to "biomass and coal combustion". That is directly related to the charcoal origin as the product of biomass pyrolysis, despite the fact the process occurs with vary limited oxygen access and cannot be considered combustion in the proper this term meaning. The W1, W11, and W18 samples show the extreme values of the diagnostic ratios and they do not fit to the whole sample set (Fig. 4).

\section{Discussion}

Taking into account the degree of risk to the environment, and above all to human health the toxic elements determined in charcoal during the analysis were divided into four groups: 
- Potentially very highly harmful elements: $\mathrm{As}, \mathrm{Cd}, \mathrm{Cu}$. $\mathrm{Hg}$ and $\mathrm{Pb}$.

- Potentially highly harmful elements: $\mathrm{Zn}, \mathrm{Ba}, \mathrm{Cr}, \mathrm{Mn}$ and Mo.

- Elements representing a lesser degree of hazard: $\mathrm{Co}, \mathrm{Ni}, \mathrm{Sn}$ and Te.

- Elements representing a low degree of hazard: $\mathrm{Ag}, \mathrm{Bi}, \mathrm{Ce}, \mathrm{Se}, \mathrm{Sr}$ and $\mathrm{Zr}$.

The contamination of charcoal with heavy metals and other toxic elements may cause their penetration into the natural environment and the human body. Skin contact with the tested grill fuel and the inhalation of coal dust occur at every stage of charcoal use, from production to use. Arsenic can enter our bodies both by inhalation and through the skin. Arsenic is a carcinogen, skin contact can cause irritation, rashes, burns and, in the case of animals. hair loss 17. Cadmium poisoning after ingestion can cause a metallic taste in the mouth, headache, fever, vomiting, diarrhea, and abdominal pain. Inhalation of cadmium leads to lung irritation, coughing, and dyspnoea. Prolonged exposure to cadmium is carcinogenic and contributes to lung and prostate cancer. Constant exposure to cadmium, even at low concentration, causes liver and kidney damage, anemia, and loss of smell18. Copper in excessive concentrations irritates the nose, throat, and eyes. Ingestion of Cu may cause headache, nausea, vomiting, diarrhea, and abdominal pain. Copper may reduce fertility, cause a greenish discoloration of the skin. hair and teeth and contribute to allergic reactions. High doses of Cu may adversely affect the liver and kidneys19. Mercury can enter our bodies both through the respiratory system and the skin. Hg irritates the throat, nose and lungs, causes a metallic taste and changes the color of the skin $\mathrm{n}$ gray. Mercury poisoning is manifested by diarrhea, nausea, vomiting, abdominal pain. Exposure to mercury vapor and skin contact may cause kidney damage, tremors, personality changes, and memory problems20. Exposure to lead has a negative impact on various internal organs including the kidneys, brain, gastrointestinal tract, hematopoietic system, and nervous system. Lead is carcinogenic; $\mathrm{Pb}$ poisoning manifests as muscle cramps and a metallic taste21. Zinc, as an element necessary for the proper functioning of the human body, is toxic in high doses. Skin contact with small fractions of $\mathrm{Zn}$ may cause skin dryness, redness and cracking. Zinc, even in small amounts, can also cause allergic reactions and eye irritation. Long-term exposure to $Z n$ causes headaches, fever, chills, difficulties in breathing, and cough22. Exposures to high concentrations of barium, especially in the form of barium salt, can cause kidney toxicity, hypertension and heart failure. On the other hand, inhalation of barium particles leads to pneumoconiosis 23 . Inhalation of chromium may cause nose bleeding, shortness of breath, wheezing, and coughing. Chromium is a skin irritant and causes allergies. It can contribute to kidney and liver damage24. In the event of excessive exposure, the toxic properties of manganese negatively affect the male reproductive organs, leading to infertility. Constant exposure to high doses of manganese leads to brain damage. Inhaling manganese leads to lung damage25. Elements $\mathrm{Co}$, Ni, Sn and Te representing a medium degree of hazard have an adverse effect on the health of living organisms when in excessive concentrations.

Co - causes skin allergies. irritates the eyes, nose and throat, has an adverse effect on the thyroid gland, liver, and lungs26.

$\mathrm{Ni}$ - irritates the skin and eyes, lungs and throat, may cause headaches and dizziness, may adversely affect the liver and kidneys27.

$\mathrm{Sn}$ - causes irritation of the skin, throat, lungs, may cause shortness of breath, vomiting, diarrhea, and may damage the liver and kidneys28.

Te - negatively affects the kidneys and nervous system and may also cause fetal damage29.

Elements $\mathrm{Ag}, \mathrm{Bi}, \mathrm{Ce}, \mathrm{Se}, \mathrm{Sr}$ and $\mathrm{Zr}$ representing a low degree of hazard to the environment and the health of living organisms can cause allergies when in excessive concentrations. Excessive exposure may lead to moderate skin and upper respiratory tract irritation and neurological - Bi30 and cardiovascular - Sr disorders. On the other hand. zirconium in concentrations above the reference values tends to accumulate in the brain31.

The sum of PAHs concentrations in the charcoal samples investigated show high variability being in the range of $12.55-3554.11 \mathrm{ng} / \mathrm{g}$ of charcoal. In the samples W16, W20, W27, W29, and W31 PAHs were absent or below a detection limit. The highest content of the PAHs sum was in the W15 and W6 samples, 3554.11 and $1156.47 \mathrm{ng} / \mathrm{g}$ of charcoal, respectively. The samples set can be divided into three sub-sets, (i) with the highest $\sum$ PAHs concentrations $>200 \mathrm{ng} / \mathrm{g}$ of charcoal comprising the W6, W7, W8, W14, W15, W21, and W26 samples (7 samples), (ii) with the intermediate $\sum$ PAHs concentrations in the range of 100$200 \mathrm{ng} / \mathrm{g}$ comprising the W10, W12, W13, W18, and W19 (5 samples), and (iii) with the lowest PAHs concentrations being < 100 ng/g comprising the W1-5, W9, W11, W17, W22, W23, W28, and W30 samples (12 samples) (Table 8).

To assess the charcoal health and environmental impact several indicators were calculated. They define the toxicity of individual PAHs and the whole compound group as well to assess the risk caused by exposure to PAHs mixture. In this approach BaP is considered to be a reference compound. The carcinogenic potential, called toxicity equivalence factor (TEF) of other PAHs is calculated relative to BaP for 16 PAHs according to the EPA list. The toxicity equivalent value (TEQ) is the sum of individual PAH concentrations and their relative toxicity coefficients32 (Table 8). Such approach is required since the carcinogenicity of individual PAH depends on its molecular structure that determines biological activity of the compound. The organization of carbon atoms as a bay region causes a high degree of biochemical reactivity to some PAHs and their metabolites33.

The mutagenicity equivalent (MEQ) was given as the sum of 16 individual PAH concentration and their relative mutagenicity coefficients34. In this approach only unsubstituted parent PAHs are considered since pyrogenic PAHs (produced under the influence of high heat) are dominated by these compounds with a slope distribution of $\mathrm{CO}>\mathrm{C} 1>\mathrm{C} 2>\mathrm{C} 3>\mathrm{C} 4$ of alkylated derivatives. Petrogenic sources (mainly crude oil, coals, and similar materials) are rich in alkyl substituted PAHs.

The TEQ and MEQ values vary considerably, since they depend on the contents of PAHs with high TEF toxicity ratios. The W15 and W6 charcoal showed the highest values; 1023.87 and 984.98 for W15 and 224.43 and 202.78, respectively (Table 8). The lowest values were found for the W3 and W17 samples, ca 2.60 for both parameters. The averages were 66.12 and 66.30 , respectively. These values suggest considerable risk for cancer and mutation caused by close contact with charcoals, both dermal and by inhalation. Comparable values of TEQ and MEQ for soot containing PAHs deriving from combustion of various solid fuels such as pellet and hard coal do not exceed 160 and 95, respectively35. 
A ratio of carcinogenic PAHs. i.e., BaA, Ch, BbF, BkF, BaP, IP, and DBA, to a sum of all PAHs ( $\sum$ PAHcarc/ $\sum$ PAHtot in Table 8 ) expresses carcinogenicity of charcoal; the closer value to 1.0 the higher carcinogenicity36-37-34. In the investigated charcoals the values of this ratio are 0.80 on average, with 15 samples, i.e., $50 \%$ of the set, exceeding these average values. The W15 and W17 samples show $\sum$ PAHcarc/ $\sum$ PAHtot above 0.90 ( 0.90 and 0.94 , respectively). Even the lowest $\sum$ PAHcarc/ $\sum$ PAHtot value is 0.59 (W1). This indicates high carcinogenicity of charcoals investigated.

BaPeq has been used to evaluate the toxicity of PAHs (Table 8). This method is typically employed by researchers to estimate BaPeq toxicity of different PAHs in air, soil, and street dust38-39-40. The ratio values varied considerably from 2.53 (W3) to 987.11 (W15). The highest level corresponds to that found in carbon black manufacturing industry41 and indicates a serious occupational risk in workers caused by exposure to charcoals. The inhalation of charcoal dust may lead to the lung cancer whereas the direct contact to various forms of skin cancers.

There is no significant correlation between the method of charcoal production, wood type, and carcinogenicity of the final product.

\section{Summary}

Charcoal samples were investigated to find their health and environmental impact. It was found that all samples were contaminated with elements representing a very high degree of hazard (e.g., $\mathrm{Cu}$ ), a high degree of hazard (Ba and $\mathrm{Mn}$ ), and a medium degree of hazard (Sr). In the group of elements with a very high degree of toxicity, their sum in seventeen samples was above $2 \mathrm{ppm}$. The four elements representing a high degree of hazard exceeded 500 ppm in total in each sample. The elements representing a medium degree of hazard exceeded the level of $2 \mathrm{ppm}$ in the next seventeen samples. The group of elements representing the lowest degree of hazard, represented by only four elements, exceeded the total level of $100 \mathrm{ppm}$. The concentrations of Cu, Ba, Mn, and $\mathrm{Sr}$ exceeded from $100 \%$ up to (in extreme cases) $2100 \%$ of the lowest acceptable safe concentrations. In addition, $\mathrm{Zr}, \mathrm{Ag}, \mathrm{Se}, \mathrm{As}, \mathrm{Cd}, \mathrm{and} \mathrm{Zn}$ occasionally exceeded acceptable values. The remaining elements, including $\mathrm{Cr}, \mathrm{Mo}, \mathrm{Hg}, \mathrm{Te}, \mathrm{Sn}, \mathrm{Ce}, \mathrm{Pb}, \mathrm{Bi}$, and $\mathrm{Co}$, were present in trace amounts in the charcoal.

Given the health risk assessment for the individual carcinogenic elements, the risk of developing cancer or other health problems is high. All charcoals showed the high carcinogenicity level caused both by PAHs and carcinogenic metals occurrence, i.e., $\mathrm{Cu}, \mathrm{Ba}$, Sr, and Mn. Since $\sum$ PAHcarc/ $\sum$ PAHtot were close to 1 , together with high TEQ and MEQ values that the contact of workers with charcoal should be limited as much as possible during all production operations and packaging. Prolonged exposure to charcoal and charcoal dust containing large quantities might cause the health problems mentioned above. This applies to employees actively involved in the production and transport of charcoal, and, to a lesser extent, also to users of this fuel.

The individual protection means are required as well as procedures limiting dermal contact and charcoal dusting to decrease charcoal particles inhalation, i.e., the lung cancer risk. The key step is to work out and introduce suitable norms for charcoal production and product controlling considering the health risk to employees and future users. Whereas there are numerous regulations concerning the contact with toxic metals there is no, up to our knowledge, the regulations concerning exposure to PAHs in charcoal. In particular, the means should be undertaken to decrease the tar content in the final product since the tar is the PAHs-bearing medium. Since users are mostly exposed to evaporating PAHs during the first stage of charcoal burning at relatively low temperatures the suitable warning should be given at the charcoal packages against smoke/dust inhalation.

Moreover, there is a high variability of toxic metals contents, PAHs concentrations and values of indices assessing possible health impact together with no clear relationship between their values for charcoal and the way of charcoal production. This suggests that every producer / charcoal batch should be analyzed for contaminants content.

\section{Declarations}

\section{Acknowledgments}

This work was supported by the PetroCoal.pl, Jaworzno, Poland

\section{Author contributions}

Zbigniew Jelonek - Data curation, Formal analysis, Writing - original draft, Writing - review \& editing

Monika Fabiańska - Data curation, Formal analysis, Writing - review \& editing

Iwona Jelonek - Data curation, Writing - review \& editing

\section{Additional information}

Competing financial interests: The authors declare no competing financial interests.

\section{References}

1. Gowlett, J. A. J., Harris, J. W. K., Walton, D. \& Wood, B. A. Early archaeological sites, hominid remains and traces of fire from Chesowanja, Kenya. Nature. nr 294, 125-129 (1981). https://www.nature.com/articles/294125a0

2. Carmody Rachel, N. \& Wrangham Richard, W. The energetic significance of cooking. Journal of Human Evolution Volume. 57 (Issue 4), $379-391$ https://doi.org/10.1016/j.jhevol.2009.02.011 (2009).

3. Maddin, R., Muhly James, D. \& Wheeler Tamara, S. 1977, How the Iron Age Began. Scientific American a division of Nature America, Inc.Vol. 237, No. 4, pp.122-131https://www.jstor.org/stable/24953968

Page 13/18 
4. Gottesman, A. 2004, Inventor of weber kettle barbecue.Chicago Tribune, https://www.chicagotribune.com/news/ct-xpm-2004-05-26-0405260116story.html

5. Plaza-Bolañosa, P., Garrido Frenich, A., Luis, J. \& Vidala, M. 2010 Polycyclic aromatic hydrocarbons in food and beverages. Analytical methods and trends Journal of Chromatography A Volume 1217, Issue 41, Pages6303-6326https://doi.org/10.1016/j.chroma.2010.07.079

6. Rutkowski, A. 1978, Advances in Smoking of Foods 1st Edition Plenary Lectures Presented at the International Symposium on Advances in Smoking of Foods. ISBN: 9781483160245 https://doi.org/10.1016/C2013-0-02920-9

7. Więk, A., Tkacz, K. \& Żywica, R. Content of polycyclic aromatic hydrocarbons (PAHs) in grilled meat products depending on fat content in raw material. Żywność. Nauka. Technologia. Jakość. 2 (87), 39-50 https://doi.org/10.15193/zntj/2013/87/039-050 (2013).

http://www.pttz.org/zyw/wyd/czas/2013,\%202(87)/04_Wiek.pdf

8. Alhamdow, A. et al. Chimney sweeps in Sweden: a questionnaire-based assessment of long-term changes in work conditions, and current eye and airway symptoms. International Archives of Occupational and Environmental Health. 90 (2), https://doi.org/10.1007/s00420-016-1186-7 (2016).

9. Ociepa-Kubicka, A. \& Ociepa, E. Toksyczne oddziaływanie metali ciężkich na rośliny, zwierzęta i ludzi. Inżynieria i Ochrona Środowiska. 15 (2), $169-180$ (2012). DOI: 10.17512/ioshttps://ios.is.pcz.pl/images/ios_repo/2012/zeszyt2/2012_nr2_5-Ociepa-Kubicka_Ociepa.pdf

10. PN-EN ISO 16967:2015-06, Solid biofuels - Determination of main elements, https://sklep.pkn.pl/pn-en-iso-16967-2015-06e.html

11. PN-EN ISO 17225-1:2014-07, Solid biofuels - Fuel specifications and grades - Part 1: General requirements. https://sklep.pkn.pl/pn-en-iso-17225-1-201407e.html

12. New Jersey Department of Health and Senior Services, Hazardous substance fact sheet. https://web.doh.state.nj.us/rtkhsfs/factsheets.aspx

13. Klavina, K. \& Blumberga, D. 2015, A comparison of different charcoal production technology outputs. Environment. Technology. Resources, Rezekne, Latvia Proceedings of the 10th International Scientific and Practical Conference. Volume II, pp. 137-140, DOI: 10.13140/RG.2.1.3822.8329

14. Simoneit, B. R. T. 1998. Biomarker PAHs in the environment. In: Neilson, A.H. (Ed.), The Handbook of Environmental Chemistry 3, Part I, PAHs and Related Compounds. Springer-Verlag, Berlin, Heidelberg, pp. 176-221 (chapter 5). ISBN 978-3-642-08286-3, DOI 10.1007/978-3-540-49697-7\$4

15. Yunker, M. B. et al. PAHs in the Fraser River basin: A critical appraisal of PAH ratios as indicators of PAH source and composition. Organic Geochemistry Volume. 33 (Issue 4), 489-515 https://doi.org/10.1016/S0146-6380(02)00002-5 (2002).

16. Charriau, A., Bodineau, L., Ouddane, B. \& Fischer, J-C. Polycyclic aromatic hydrocarbons and n-alkanes in sediments of the Upper Scheldt River Basin: contamination levels and source apportionment. J. Environ. Monit. 11, 1086-1093 https://doi.org/10.1039/b819928k (2009). https://pubs.rsc.org/en/content/articlelanding/2009/EM/b819928k\#!divAbstract

17. Saha, J. C., Dikshit, A. K., Bandyopadhyay, M. \& Saha, K. C. 1999. A Review of Arsenic Poisoning and its Effects on Human Health. Critical Reviews in Environmental Science and Technology, Volume 29, Issue 3 ISSN:1064-3389DOI: 10.1080/10643389991259227

18. Sharma, H., Rawal, N. \& Mathew, B. B. The characteristics, toxicity and effects of cadmium. International Journal of Nanotechnology and Nanoscience. Vol. 3, 1-9 (2015). https://www.researchgate.net/profile/Blessy-Mathew-

4/publication/305778858_The_characteristics_toxicity_and_effects_of_cadmium/links/57a19fe508aeb1604832e9d9/The-characteristics-toxicity-andeffects-of-cadmium.pdf

19. Kumar, J., Inchulkar, S. R. \& Bhagat, S. Copper Toxicity. European Journal of Pharmaceutical and Medical Research. 5 (11), 232-2372394 (2018).

20. Kim, S. K. P. K. H., Kang, C. H., Jung, M. C. \& Yoon., H. 2009. BBQ charcoal as an important source of mercury emission. Journal of Hazardous Materials Volume 162, Issue 1, 15 February 2009, Pages 536-538 https://doi.org/10.1016/j.jhazmat.2008.05.050

21. Newton, C. et al. 1979. Lead poisoning: A comprehensive review and report of a case. Oral Surgery, Oral Pathology Volume 47, Issue 6, Pages 500-512 https://doi.org/10.1016/0030-4220(79)90271-8

22. Fosmire, G. J. 1990. Zinc toxicity. The American Journal of Clinical Nutrition, Volume 51, Issue 2, Pages225-227, https://doi.org/10.1093/ajcn/51.2.225

23. Oskarsson, A. 2015. Barium in: Handbook on the Toxicology of Metals (Fourth Edition) Volume II, Pages 625-634 Chap. 29 https://doi.org/10.1016/B978-0-444-59453-2.00029-9

24. Langård, S. \& Costa, M. 2015. Chromium in: Handbook on the Toxicology of Metals (Fourth Edition) Volume II, Pages 717-742 Chap. 33 https://doi.org/10.1016/B978-0-444-59453-2.00033-0

25. Stefanie, L. O'Neal \& Wei Zheng 2015. Manganese Toxicity Upon Overexposure: a Decade in Review. Current Environmental Health Reports volume 2, pages 315-328. https://link.springer.com/article/10.1007/s40572-015-0056-x

26. Lison, D. 2015. Cobalt in: Handbook on the Toxicology of Metals (Fourth Edition) Volume II, Pages 743-763 Chap. 34 https://doi.org/10.1016/B978-0444-59453-2.00034-2

27. Klein, C. \& Costa, M. 2015. Nickel in: Handbook on the Toxicology of Metals (Fourth Edition) Volume II, Pages 1091-1111 Chap. 48 https://doi.org/10.1016/B978-0-444-59453-2.00048-2

28. Ostrakhovitch, E. A. 2015. Tin: Handbook on the Toxicology of Metals (Fourth Edition) Volume II, Pages1241-1285Chap. 56 https://doi.org/10.1016/B978-0-444-59453-2.00056-1

29. Gerhardsson, L. 2015. Tellurium: Handbook on the Toxicology of Metals (Fourth Edition) Volume II, Pages1217-1228Chap. 54 https://doi.org/10.1016/B978-0-444-59453-2.00054-8

30. Fowler, B. A., Sullivan, D. W. \& Sexton, M. J. 2015. Bismuth: Handbook on the Toxicology of Metals (Fourth Edition) Volume II, Pages655666https://doi.org/10.1016/B978-0-444-59453-2.00031-7

31. Ghosh, S., Sharma, A. \& Talukder, G. Zirconium An abnormal trace element in biology. Biological Trace Element Research volume. 35, 247-271 https://doi.org/10.1007/BF02783770 (1992).

Page $14 / 18$ 
32. Nisbet, I. C. T. \& LaGoy, P. K. 1992. Toxic equivalency factors (TEFs) for polycyclic aromatic hydrocarbons (PAHs). Regulatory Toxicology and Pharmacology Volume 16, Issue 3, Pages290-300https://doi.org/10.1016/0273-2300(92)90009-X

33. Zhang, D. et al. Source identification and health risk of polycyclic aromatic hydrocarbons associated with electronic dismantling in Guiyu town, South China. Journal of Hazardous Materials Volume. 192 (Issue 1), 151-157 https://doi.org/10.1016/j.jhazmat.2011.03.109 (2011).

34. Rogula-Kozłowska, W., Kozielska, B. \& Klejnowski, K. 2013. Concentration, Origin and Health Hazard from Fine Particle-Bound PAH at Three Characteristic Sites in Southern Poland. Bulletin of Environmental Contamination and Toxicology volume 91, pages349-355https://doi.org/10.1007/s00128-013-10601

35. Szatyłowicz, E. \& Skoczko, I. 2019. Evaluation of the PAH Content in Soot from Solid Fuels Combustion in Low Power Boilers. Energies 2019 , 12 , 4254 https://doi.org/10.3390/en12224254

36. Bourotte, C., Fort, M. C., Taniguchi, S., Bícego, M. C. \& Lotufo, P. A. A wintertime study of PAHs in fine and coarse aerosols in São Paulo city, Brazil. Atmospheric Environment Volume. 39, Issue 21, Pages 3799-3811 https://doi.org/10.1016/j.atmosenv.2005.02.054 (2005).

37. del Rosario Sienra, M., Rosazza, N. G. \& Préndez Bolivar, M. M. Polycyclic aromatic hydrocarbons and their molecular diagnostic ratios in urban atmospheric respirable particulate matter. Atmospheric Research Volume. 75 (Issue 4), 267-281 https://doi.org/10.1016/j.atmosres.2005.01.003 (2005).

38. Barrán-Berdón, A. L. et al. Verduzco Cuéllar B. 2012. Polycyclic aromatic hydrocarbons in soils from a brick manufacturing location in central Mexico.Revista Internacional de Contaminacion Ambiental28 (4),277-288

39. Qi, H. et al. Concentrations and Sources of Polycyclic Aromatic Hydrocarbons in Indoor Dust in China. Science of the Total Environment. 491-492, 100107 https://doi.org/10.1016/j.scitotenv.2014.01.119 (2014).

40. Wei, H., Guangbin, L., Yong, T. \& Qin, Z. Emission of polycyclic aromatic hydrocarbons from different types of motor vehicles' exhaust. Environmental Earth Sciences volume. 74, 5557-5564 https://doi.org/10.1007/s12665-015-4570-9 (2015).

41. Tsai, P. J., Shieh, H. Y., Lee, W. J. \& Lai, S. O. 2001. Health-risk assessment for workers exposed to polycyclic aromatic hydrocarbons (PAHs) in a carbon black manufacturing industry. Science of The Total Environment Volume 278, Issues 1-3, 20, Pages 137-150 https://doi.org/10.1016/S00489697(01)00643-X

\section{Figures}

\section{naphthalene}

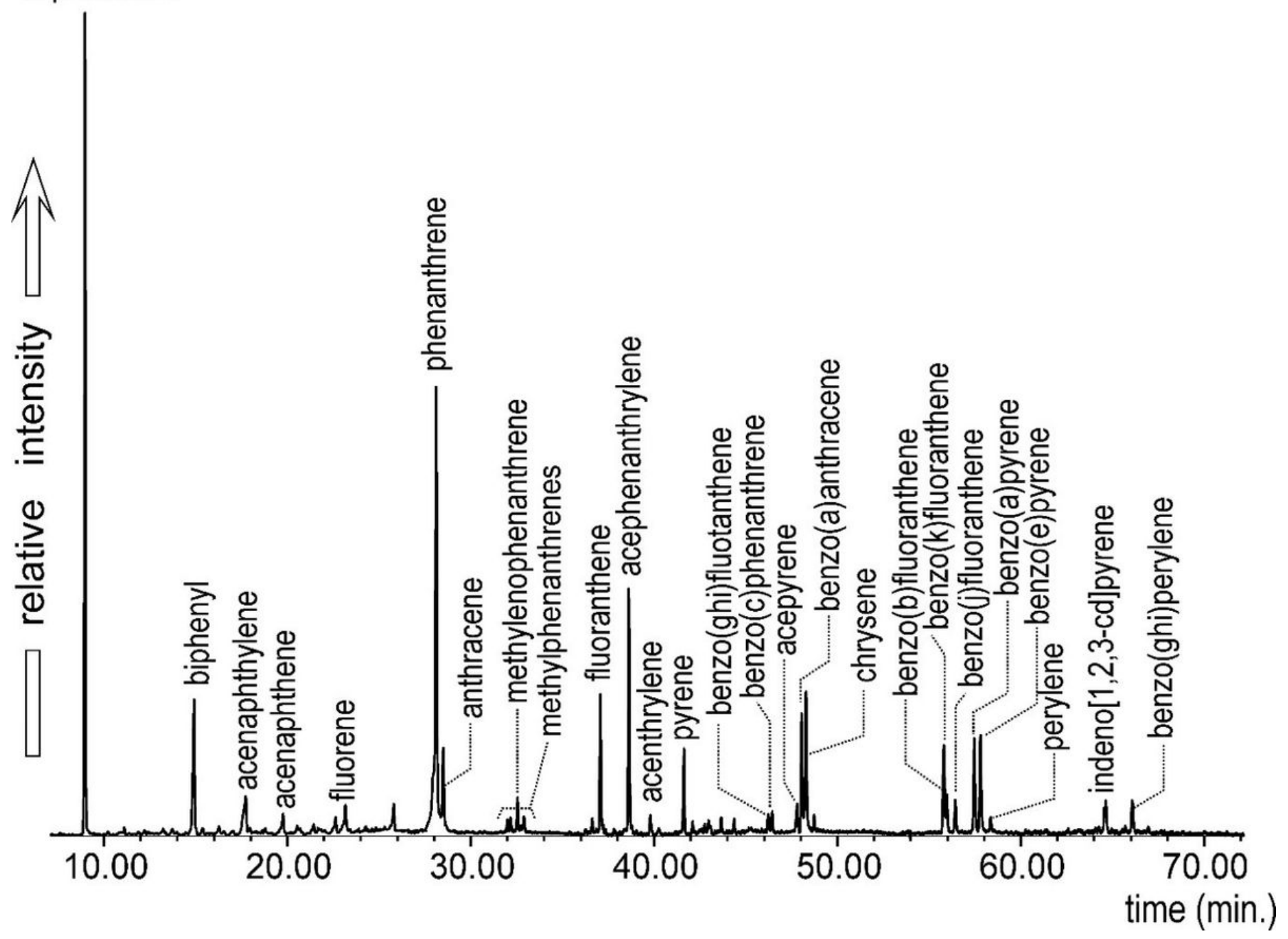

Page 15/18 
Figure 1

Generalized PAHs distribution in charcoal extract (sample W7)

4-ring PAHs

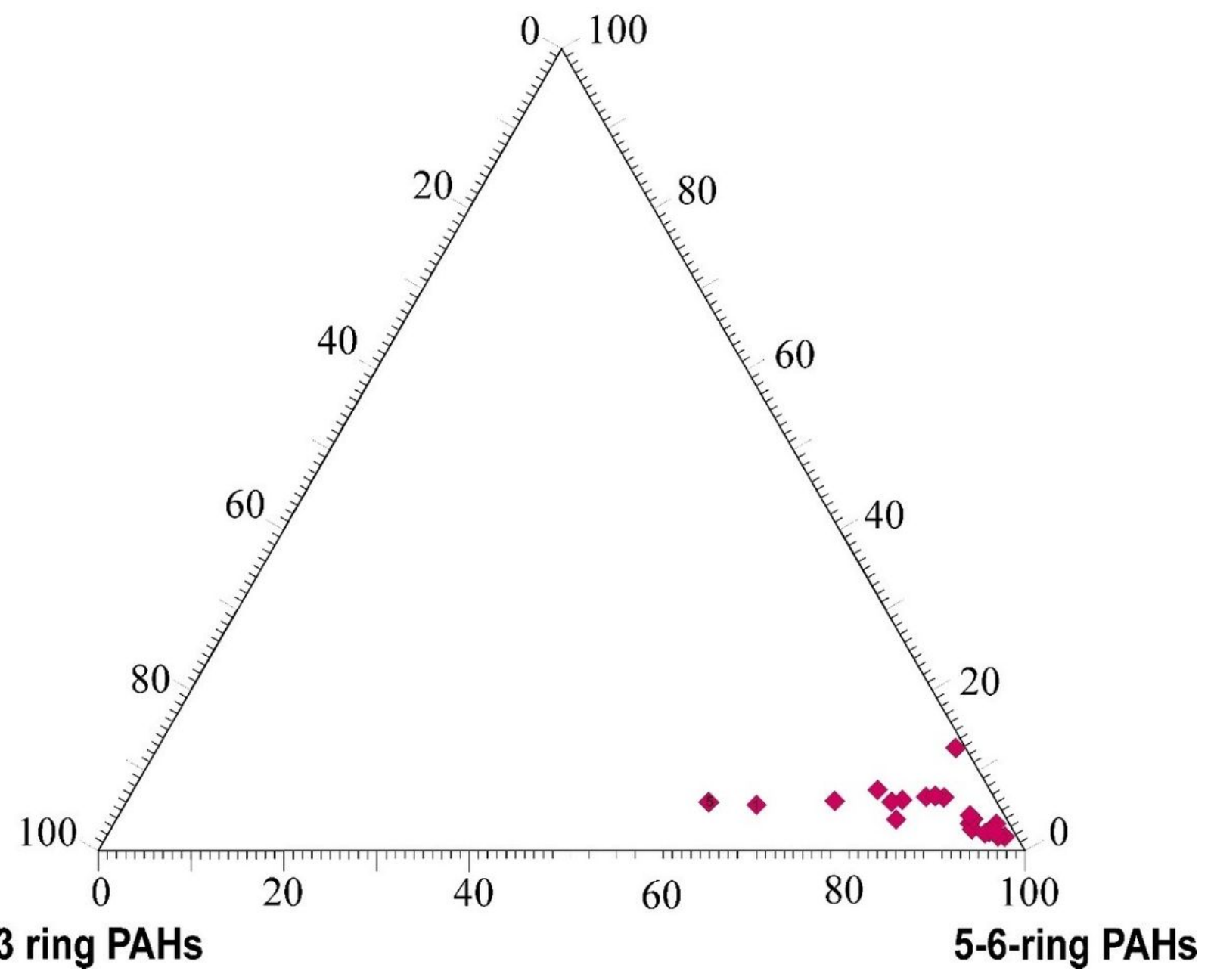

Figure 2

Distribution of PAHs according to number of rings in a molecule 

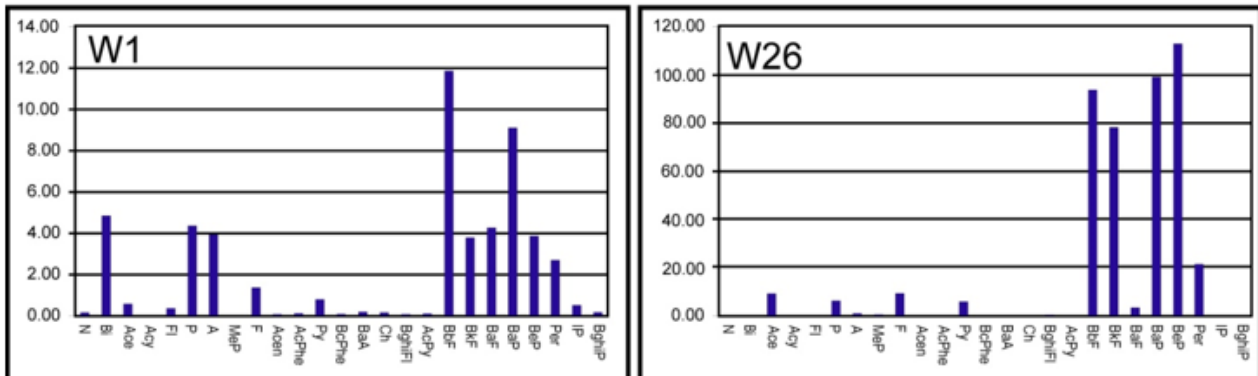

lighter PAHs almost absent

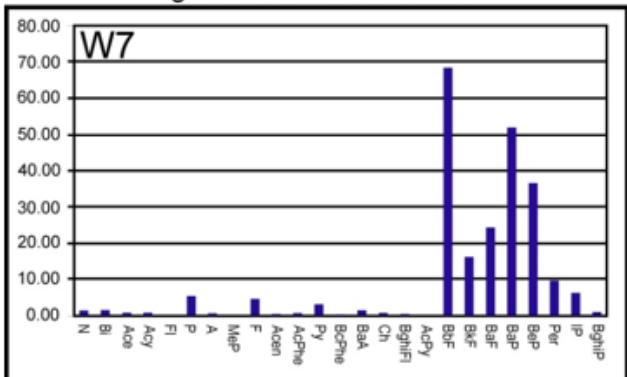

benzopyrenes dominate over benzofluoranthenes

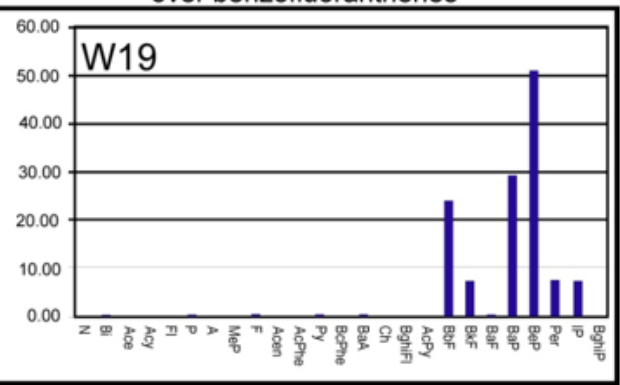

Figure 3

Distribution types of PAHs in charcoal extracts
petroleum biomass and
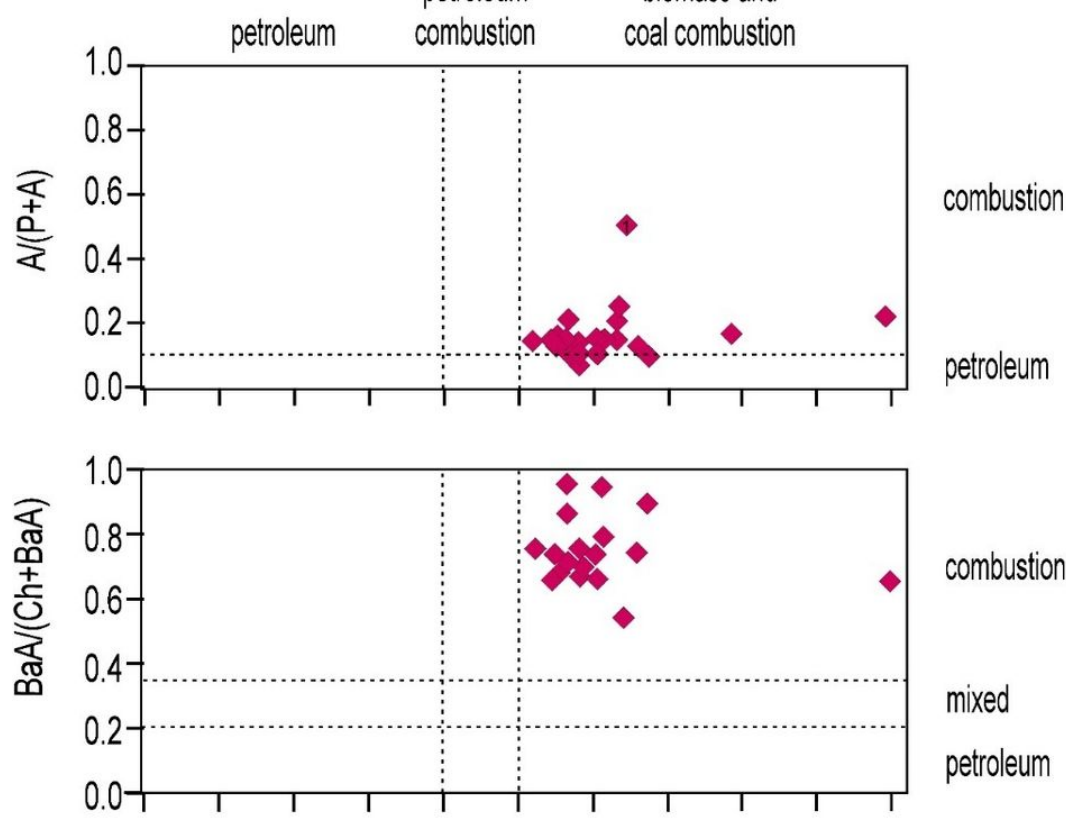

combustion

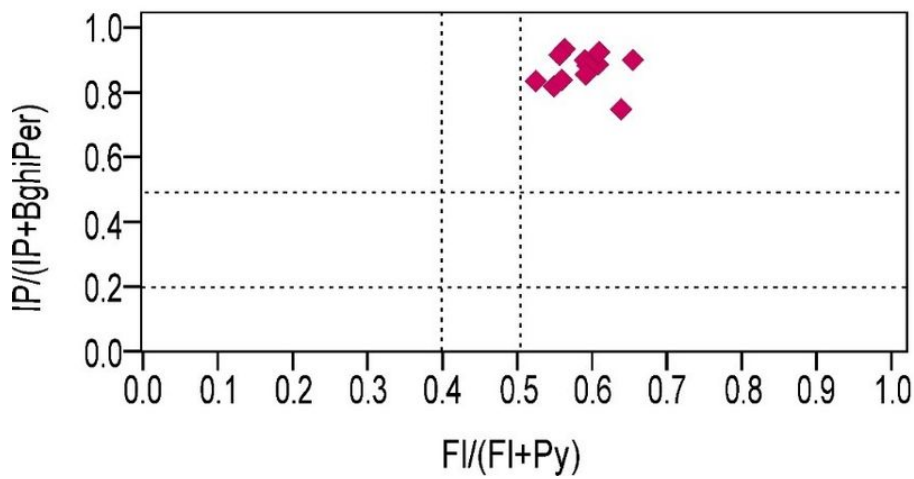

biomass and coal

combustion

petroleum

combustion

petroleum 
Figure 4

Diagnostics diagrams of PAHs in charcoals investigated15 A - anthracene, $\mathrm{P}$ - phenanthrene, $\mathrm{FI}$ - fluoranthene, Py - pyrene, BaA - benzo(a)anthracene, Ch chrysene, IP - indeno[1,2,3-cd]pyrene, BghiP - benzo(ghi)perylene 